\title{
Vanishing superconformal indices and the chiral symmetry breaking
}

\author{
V.P. Spiridonov ${ }^{a, 1}$ and G.S. Vartanov ${ }^{b}$ \\ ${ }^{a}$ Bogoliubov Laboratory of Theoretical Physics, JINR, \\ J. Curie str. 6, Dubna, Moscow Region 141980, Russia \\ ${ }^{b}$ DESY Theory, \\ Notkestrasse 85, 22603 Hamburg, Germany \\ E-mail: spiridon@theor.jinr.ru, vartanovg@yahoo.com
}

Abstract: Superconformal indices of $4 d \mathcal{N}=1 \mathrm{SYM}$ theories with $\mathrm{SU}(N)$ and $\operatorname{SP}(2 N)$ gauge groups are investigated for $N_{f}=N$ and $N_{f}=N+1$ flavors, respectively. These indices vanish for generic values of the flavor fugacities. However, for a singular submanifold of fugacities they behave like the Dirac delta functions and describe the chiral symmetry breaking phenomenon. Similar picture holds for partition functions of $3 d$ supersymmetric field theories with the chiral symmetry breaking.

KEYWords: Supersymmetric gauge theory, Supersymmetry and Duality, Confinement

ARXIV EPRINT: 1402.2312

\footnotetext{
${ }^{1}$ Current address: Max-Planck-Institut für Mathematik, Vivatsgasse 7, 53111 Bonn, Germany.
} 


\section{Contents}

1 Introduction 1

2 Superconformal index $\quad 2$

3 Chiral symmetry breaking for $G_{c}=\mathrm{SU}(2) \quad 5$

$4 \mathrm{SU}(N), N>2$, gauge group case $\quad 8$

4.1 Breaking to the diagonal subgroup: $\mathrm{SU}(N)_{l} \times \mathrm{SU}(N)_{r} \rightarrow \mathrm{SU}(N)_{d}$

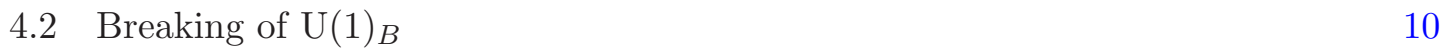

5 Proofs $\quad 11$

$5.1 N_{f}=N=2$ case $\quad 11$

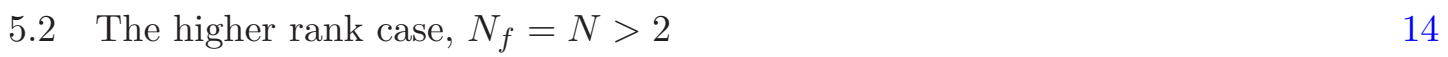

$\begin{array}{lll}5.3 & \text { The case } N_{f}<N & 17\end{array}$

6 Chiral symmetry breaking for $G_{c}=S P(2 N) \quad 18$

$\begin{array}{lll}7 & 3 d \text { theories with chiral symmetry breaking } & 22\end{array}$

8 Conclusion $\quad 25$

\section{Introduction}

We take as a starting point the remarkable observation of [1] that superconformal indices (SCIs) of $4 d$ supersymmetric field theories are expressed in terms of elliptic hypergeometric integrals (EHIs) discovered in [2,3] (for a review see [4]). SCIs were introduced in [5] and $[6,7]$ from different physical motivations. They describe also indices of nonconformal supersymmetric field theories on curved backgrounds flowing to a superconformal infrared fixed point [8]. In [5] the main target was the AdS/CFT correspondence. In [6, 7] BPS operators of $\mathcal{N}=1 \mathrm{SYM}$ theories were studied and the equality of SCIs for Seiberg dual theories was conjectured. In [1] this hypothsis was proven analytically for the initial Seiberg duality [9] using mathematical properties of EHIs established in [2, 3, 10, 11]. Following this result we systematically considered the connection of $\mathcal{N}=1$ supersymmetric field theories with the theory of EHIs [12-18] We showed that available physical checks for Seiberg dualities can be described by known general properties of EHIs, conjectured many new mathematical identities and found many new physical dualities. SCI techniques was applied also to the description of $S$-dualities of $\mathcal{N}=2,4$ extended supersymmetric field theories in [19-21] and [16]. 
In [14] it was conjectured that all 't Hooft anomaly matching conditions are related to the total ellipticity condition for EHIs [4]. As shown in [22] this is not so for $\mathrm{U}(1)_{R}$ and $\mathrm{U}(1)_{R}^{3}$-anomalies. However, in [18] it was demonstrated that all anomaly matchings for Seiberg dual theories follow from $\mathrm{SL}(3, \mathbb{Z})$-modular transformation properties of the kernels of dual SCIs. One can consider modifications of SCIs such as the addition of charge conjugation [23], inclusion of surface operators [24, 25] or line operators [26-28], etc. Connection of SCIs of $4 d$ theories and partition functions of $2 d$ statistical mechanics models was discussed in [29]. An interesting $5 d / 4 d$ boundary field theory with the extended $E_{7}$-flavor symmetry was proposed in [30], which is based on the particular $4 d$ multiple dual theories [12] and $W\left(E_{7}\right)$-symmetry of corresponding SCIs [3, 10]. A similar interpretation for SCIs with $W\left(E_{6}\right)$-symmetry [12] was proposed in [31].

In this paper we would like to discuss a particular phenomenon for $\mathcal{N}=1 \mathrm{SYM}$ theories pointed out in [32] which is known as the confinement with chiral symmetry breaking when the global symmetry group gets broken. Originally such a physical effect was considered for $\mathrm{SU}(N)$-gauge group supersymmetric quantum chromodynamics with $N_{f}=N$ and it should be contrasted to the so-called s-confinement occurring at $N_{f}=N+1$ (i.e., the confinement without breaking global symmetries). Later the theories with quantum modified moduli space were systematically studied in [34, 35]. SCIs of such theories are not well defined, in particular, they were not computed from the first principles using the localization techniques. The formal free field computations yield diverging results (actually, such computations are not affected even by the anomalies $[15,18])$. From mathematical point of view the relevant behavior of SCIs was partially considered for $N=2$ in [36]. We compute SCIs of such theories in general case $N_{f}=N$ and show that they involve Dirac delta functions reflecting the presence of chiral symmetry breaking. Similar considerations are fulfilled for a $4 d$ theory with $S P(2 N)$ gauge group and some $3 d$ theories.

\section{Superconformal index}

Superconformal index counts BPS states protected by one supercharge which cannot be combined to form long multiplets. The $\mathcal{N}=1$ superconformal algebra of space-time symmetry group $\mathrm{SU}(2,2 \mid 1)$ is generated by $J_{i}, \bar{J}_{i}$ (Lorentz rotations), $P_{\mu}$ (translations), $K_{\mu}$, (special conformal transformations), $H$ (dilatations) and $R\left(\mathrm{U}(1)_{R}\right.$-rotations). There are also four supercharges $Q_{\alpha}, \bar{Q}_{\dot{\alpha}}$ and their superconformal partners $S_{\alpha}, \bar{S}_{\dot{\alpha}}$. Distinguishing a pair of supercharges, say, $Q=\bar{Q}_{1}$ and $Q^{\dagger}=-\bar{S}_{1}$, one has

$$
\left\{Q, Q^{\dagger}\right\}=2 \mathcal{H}, \quad Q^{2}=\left(Q^{\dagger}\right)^{2}=0, \quad \mathcal{H}=H-2 \bar{J}_{3}-3 R / 2 .
$$

The SCI is defined now by the gauge invariant trace

$$
I(p, q, \underline{y})=\operatorname{Tr}\left((-1)^{\mathcal{F}} p^{\mathcal{R} / 2+J_{3}} q^{\mathcal{R} / 2-J_{3}} \prod_{k} y_{k}^{F_{k}} e^{-\beta \mathcal{H}}\right), \quad \mathcal{R}=R+2 \bar{J}_{3},
$$

where $\mathcal{F}$ is the fermion number operator. Parameters $p$ and $q$ are fugacities for the operators $\mathcal{R} / 2 \pm J_{3}$ commuting with $Q$ and $Q^{\dagger} . F_{k}$ are the maximal torus generators of the flavor 


\begin{tabular}{|c|c|c|c|c|c|}
\hline & $\mathrm{SU}(N)$ & $\mathrm{SU}(N+1)_{l}$ & $\mathrm{SU}(N+1)_{r}$ & $\mathrm{U}(1)_{B}$ & $\mathrm{U}(1)_{R}$ \\
\hline$Q$ & $f$ & $f$ & 1 & 1 & $\frac{1}{N+1}$ \\
$\widetilde{Q}$ & $\bar{f}$ & 1 & $\bar{f}$ & -1 & $\frac{1}{N+1}$ \\
$V$ & $a d j$ & 1 & 1 & 0 & 1 \\
\hline
\end{tabular}

Table 1. Matter content of the electric theory.

group $F$ with the corresponding fugacities $y_{k}$. Since relation (2.1) is preserved by the operators used in (2.2) only zero modes of the operator $\mathcal{H}$ contribute to the trace.

An explicit computation of SCIs for $\mathcal{N}=1$ theories results in the prescription $[1,6,7]$ according to which one first composes the single particle states index

$$
\begin{aligned}
& \operatorname{ind}(p, q, \underline{z}, \underline{y})=\frac{2 p q-p-q}{(1-p)(1-q)} \chi_{a d j, G}(\underline{z}) \\
& \quad+\sum_{j} \frac{(p q)^{R_{j} / 2} \chi_{R_{F}, j}(\underline{y}) \chi_{R_{G}, j}(\underline{z})-(p q)^{1-R_{j} / 2} \chi_{\bar{R}_{F}, j}(\underline{y}) \chi_{\bar{R}_{G}, j}(\underline{z})}{(1-p)(1-q)} .
\end{aligned}
$$

The contribution of gauge superfields lying in the adjoint representation of the gauge group $G_{c}$ is described by the first term in (2.3). The sum over $j$ corresponds to the contribution of chiral matter superfields $\Phi_{j}$ transforming as the gauge group representations $R_{G, j}$ and flavor symmetry group representations $R_{F, j}$. The functions $\chi_{a d j}(\underline{z}), \chi_{R_{F}, j}(\underline{y})$ and $\chi_{R_{G}, j}(\underline{z})$ are the corresponding characters and $R_{j}$ are the field $R$-charges. The variables $z_{1}, \ldots, z_{\text {rank }} G_{c}$ are the maximal torus fugacities of $G_{c}$. To obtain the full SCI the function $\operatorname{ind}(p, q, \underline{z}, \underline{y})$ is inserted into the "plethystic" exponential which is averaged over the gauge group. This yields the following matrix integral

$$
I(p, q, \underline{y})=\int_{G_{c}} d \mu(\underline{z}) \exp \left(\sum_{n=1}^{\infty} \frac{1}{n} \operatorname{ind}\left(p^{n}, q^{n}, \underline{z}^{n}, \underline{y}^{n}\right)\right),
$$

where $d \mu(\underline{z})$ is the $G_{c}$-invariant measure. This formula has no rigorous mathematical justification and the region of its applicability is not completely determined. In $[6,7]$ it was derived basically from the free field theory without taking into account possible complicated dynamical effects. Therefore formally it can be applied even to the anomalous theories.

Let us consider an example of the $s$-confining theory from [32]. Namely, take a $4 d$ $\mathcal{N}=1$ SYM theory with $G_{c}=\mathrm{SU}(N)$ gauge group and $\mathrm{SU}\left(N_{f}\right)_{l} \times \mathrm{SU}\left(N_{f}\right)_{r} \times \mathrm{U}(1)_{B}$ flavor symmetry group and $N_{f}=N+1$. The original (electric) theory has $N+1$ left and $N+1$ right quarks $Q$ and $\widetilde{Q}$ lying in the fundamental and antifundamental representations of $\mathrm{SU}(N)$. They have +1 and -1 baryonic charges and the $R$-charge $R=1 /(N+1)$. The field content of the described theory is summarized in table 1. The general Seiberg duality [9] is supposed to live in the conformal window $3 N / 2<N_{f}<3 N$, and we see that the duality we consider lies outside of it.

SCI of this ("electric") theory is given by the following EHI [1]:

$$
I_{E}=\kappa_{N} \int_{\mathbb{T}^{N-1}} \frac{\prod_{i=1}^{N+1} \prod_{j=1}^{N} \Gamma\left(s_{i} z_{j}, t_{i}^{-1} z_{j}^{-1} ; p, q\right)}{\prod_{1 \leq i<j \leq N} \Gamma\left(z_{i} z_{j}^{-1}, z_{i}^{-1} z_{j} ; p, q\right)} \prod_{j=1}^{N-1} \frac{d z_{j}}{2 \pi \mathrm{i} z_{j}},
$$




\begin{tabular}{|c|c|c|c|c|}
\hline & $\mathrm{SU}(N+1)_{l}$ & $\mathrm{SU}(N+1)_{r}$ & $\mathrm{U}(1)_{B}$ & $\mathrm{U}(1)_{R}$ \\
\hline$M$ & $f$ & $\bar{f}$ & 0 & $\frac{2}{N+1}$ \\
$B$ & $\bar{f}$ & 1 & $N$ & $\frac{N}{N+1}$ \\
$\widetilde{B}$ & 1 & $f$ & $-N$ & $\frac{N}{N+1}$ \\
\hline
\end{tabular}

Table 2. Matter content of the magnetic theory.

where $\mathbb{T}$ denotes the unit circle with positive orientation, $\prod_{j=1}^{N} z_{j}=1,\left|s_{i}\right|,\left|t_{i}^{-1}\right|<1$, and the balancing condition reads $S T^{-1}=p q$ with $S=\prod_{i=1}^{N+1} s_{i}, T=\prod_{i=1}^{N+1} t_{i}$. Here we introduced the parameters $s_{i}$ and $t_{i}$ as

$$
s_{i}=(p q)^{R / 2} v x_{i}, \quad t_{i}=(p q)^{-R / 2} v y_{i},
$$

where $v, x_{i}$ and $y_{i}$ are fugacities for $\mathrm{U}(1)_{B}, \mathrm{SU}(N+1)_{l}$ and $\mathrm{SU}(N+1)_{r}$ groups, respectively, with the constraints $\prod_{i=1}^{N+1} x_{i}=\prod_{i=1}^{N+1} y_{i}=1$, and

$$
\kappa_{N}=\frac{(p ; p)_{\infty}^{N-1}(q ; q)_{\infty}^{N-1}}{N !}, \quad(a ; q)_{\infty}=\prod_{k=0}^{\infty}\left(1-a q^{k}\right) .
$$

We use also conventions

$$
\Gamma(a, b ; p, q):=\Gamma(a ; p, q) \Gamma(b ; p, q), \quad \Gamma\left(a z^{ \pm 1} ; p, q\right):=\Gamma(a z ; p, q) \Gamma\left(a z^{-1} ; p, q\right),
$$

where

$$
\Gamma(z ; p, q)=\prod_{i, j=0}^{\infty} \frac{1-z^{-1} p^{i+1} q^{j+1}}{1-z p^{i} q^{j}}, \quad|p|,|q|<1,
$$

is the (standard) elliptic gamma function.

According to [32] the dual ("magnetic") theory is described by colorless mesons and baryons, i.e. the dual theory has no gauge group, but it has the same flavor symmetry. Its description is given in terms of baryons $B$ and $\widetilde{B}$ with $\mathrm{U}(1)_{B}$-charges $N$ and $-N$ and the $R$-charges $N /(N+1)$. There are also mesons of $R$-charge $2 /(N+1)$ lying in the fundamental representation of $\mathrm{SU}(N+1)_{l}$ and antifundamental representation of $\mathrm{SU}(N+1)_{r}$ $\left(M_{i}^{j}=Q_{i} \widetilde{Q}^{j}, i, j=1, \ldots, N+1\right)$. We collect all fields data in table 2 .

The SCI of the magnetic theory is

$$
I_{M}=\prod_{1 \leq i, j \leq N+1} \Gamma\left(s_{i} t_{j}^{-1} ; p, q\right) \prod_{i=1}^{N+1} \Gamma\left(S s_{i}^{-1}, T^{-1} t_{i} ; p, q\right) .
$$

As discovered in [1], the equality of SCIs $I_{E}=I_{M}$ coincides with the mathematical identity initially established for $N=2$ in [2] as the evaluation formula for an elliptic beta integral and conjectured for general $N$ in [3] and proven completely in [10, 11].

Following Seiberg [32] one can integrate out one flavor and come to supersymmetric quantum chromodynamics theory with $N_{f}=N$ when the classical moduli space is modified 
at the quantum level leading to the chiral symmetry breaking. From the SCI point of view the condition of integrating out a flavor is expressed by the following constraint on fugacities

$$
s_{N+1} t_{N+1}^{-1}=p q .
$$

Substituting this restriction into (2.5) and using the reflection property

$$
\Gamma\left(z, \frac{p q}{z} ; p, q\right)=1
$$

we see that the gamma functions involving parameters $s_{N+1}$ and $t_{N+1}$ disappear, while the expression for the dual theory (2.8) seems to vanish, since $\Gamma(p q ; p, q)=0$. However, this is true only for generic values of parameters $s_{i}$ and $t_{i}, i=1, \ldots, N$, and a more accurate analysis of the corresponding SCIs should be carried out for special values of the fugacities. Namely, if $s_{i} t_{j}^{-1} \rightarrow 1$ for some $i$ and $j$, then $\Gamma\left(s_{i} t_{j}^{-1} ; p, q\right)$ diverges and we have two competing regimes. Resolution of the emerging uncertainty can lead to a non-zero answer. The naive prescription for building SCIs (2.3) and (2.4) does not apply in such cases.

\section{Chiral symmetry breaking for $G_{c}=\mathrm{SU}(2)$}

We start our analysis of SCIs for the case of $4 d \mathcal{N}=1$ SYM theory with $\mathrm{SU}(2)$ gauge group and four quark fields $\left(N_{f}=2\right)$ considered in [32]. As we will see, SCIs vanish for generic values of fugacities and in some special cases they have delta function type singularities.

Let us take four parameters $s_{j} \in \mathbb{T}$ subject to the balancing constraint $\prod_{j=1}^{4} s_{j}=1$. In the parametrization $s_{j}=e^{2 \pi \mathrm{i} \phi_{j}}, 0 \leq \phi_{j}<1$, one has $\sum_{j=1}^{4} \phi_{j}=0(\bmod 1)$. Denote as $\mathbb{T}_{d}$ an infinitesimal deformation of the unit circle with positive orientation such that the points $s_{j}$ lie inside $\mathbb{T}_{d}$ and the points $s_{j}^{-1}$ are outside $\mathbb{T}_{d}$. Particular values of $s_{j}$ when such a contour does not exist represent a special interest and they will be treated through a limiting procedure. For this set of parameters we define the integral

$$
I_{E}=\frac{(p ; p)_{\infty}(q ; q)_{\infty}}{2} \int_{\mathbb{T}_{d}} \frac{\prod_{j=1}^{4} \Gamma\left(s_{j} z^{ \pm 1} ; p, q\right)}{\Gamma\left(z^{ \pm 2} ; p, q\right)} \frac{d z}{2 \pi \mathrm{i} z} .
$$

Our aim is to show that for arbitrary values of parameters $s_{j}$ (excluding the cases when $s_{j}=s_{k}$ for $j \neq k$ ) one can evaluate this integral and come to the equality $I_{E}=I_{M}$ with

$$
I_{M}=\frac{1}{(p ; p)_{\infty}(q ; q)_{\infty}} \sum_{j=2}^{4} \Gamma\left(s_{1} s_{k}, s_{1} s_{l}, s_{j} s_{k}, s_{j} s_{l} ; p, q\right) \delta\left(\phi_{1}+\phi_{j}\right),
$$

where the triple $(j, k, l)$ is a cyclic permutation of $(2,3,4)$ and $\delta(\phi)$ is the periodic Dirac delta function with period $1, \delta(\phi+1)=\delta(\phi)$. There are many equivalent forms of $I_{M}$, e.g.

$$
I_{M}=\Gamma\left(s_{1}^{ \pm 1} s_{2}^{ \pm 1} ; p, q\right) \frac{\delta\left(\phi_{1}+\phi_{3}\right)+\delta\left(\phi_{1}+\phi_{4}\right)}{(p ; p)_{\infty}(q ; q)_{\infty}}+\Gamma\left(s_{2}^{ \pm 1} s_{3}^{ \pm 1} ; p, q\right) \frac{\delta\left(\phi_{1}+\phi_{2}\right)}{(p ; p)_{\infty}(q ; q)_{\infty}} .
$$

It can be checked that $I_{M}$ is symmetric in parameters $s_{j}$ due to the balancing condition $\prod_{j=1}^{4} s_{j}=1$, although this is not apparent. The equality $I_{E}=I_{M}$ can be obtained by 
taking accurate limits of parameters in the elliptic beta integral which will be described below. We observe from the above relation that for generic values of $s_{j}, j=1, \ldots, 4$, expressions $I_{E}$ and $I_{M}$ vanish and only for the cases when $s_{j} s_{k}=1$ for some $j \neq k$ one has a non-trivial result.

Univariate functions determined by Cauchy type integrals are holomorphic except of the values of the arugument lying on the integration contour. Corresponding simple pole singularities lead to branch cuts and the function may have different values for the argument approaching the integration contour from one or another side as described by the Sokhotsky-Plemelj formulas [33]. Different singular kernels can lead to more complicated integral singularities. Positions of zeros and poles of elliptic gamma functions indicate that the integral (3.1) defines a holomorphic function of parameters for $\left|s_{j}\right|<1$. As follows from general considerations of [10] the product of this integral with the function $\prod_{1 \leq i<j \leq 4} \prod_{k, l=0}^{\infty}\left(1-s_{i} s_{j} p^{k} q^{l}\right)$ is a holomorphic function for all values of $s_{j}$. This means that the integral may be singular only for domains of $s_{j}$ determined by zero locus of the indicated multiplier. Our aim is to determine the nature of such singularities for $s_{i} s_{j}=1$, $i \neq j$, and show that they are described by Dirac delta functions.

To make formulas (3.1) and (3.2) a little more transparent and lucid, let us assume that we deal with the singular manifold for delta function $\delta\left(\phi_{1}+\phi_{3}\right)$. This means that $s_{1} s_{3}=1$ which also implies that $s_{2} s_{4}=1$ because of the balancing condition. As a result, one has in the numerator of the integrand of $I_{E}$ the following expression

$$
\Gamma\left(s_{1}^{ \pm 1} z^{ \pm 1}, s_{2}^{ \pm 1} z^{ \pm 1} ; p, q\right),
$$

and the coefficient depending only on elliptic gamma functions in $I_{M}$ has the form

$$
\Gamma\left(s_{1}^{ \pm 1} s_{2}^{ \pm 1} ; p, q\right) .
$$

The structure of these products of elliptic gamma functions suggests the physical meaning of the above identity $I_{E}=I_{M}$ as the equality of SCIs for the taken theory with the chiral symmetry breaking and its dual.

So, the $4 d \mathcal{N}=1$ SYM theory with $\mathrm{SU}(2)$ gauge group and two (left and right) flavors has a naive $\mathrm{SU}(4)$ flavor symmetry group. The gauge invariant combinations of chiral fields are

$$
V^{i j}=Q^{i} Q^{j}
$$

where $Q^{i}, i=1, \ldots, 4$, are chiral superfields in the fundamental representation of the gauge group $\mathrm{SU}(2)$. They are restricted at the classical level by the following relation

$$
\epsilon_{i_{1} i_{2} i_{3} i_{4}} V^{i_{1} i_{2}} V^{i_{3} i_{4}}=0 .
$$

At the quantum level this restriction is deformed and becomes the nonzero pfaffian constraint

$$
\operatorname{Pf} V=\Lambda^{4}
$$

where $\Lambda$ is some characteristic energy scale. This scale breaks the conformal symmetry and, so, the term "superconformal index" is misleading in this case, i.e. it can be called 


\begin{tabular}{|c|c|c|c|}
\hline & $\mathrm{SU}(2)$ & $S P(4)$ & $\mathrm{U}(1)_{R}$ \\
\hline$Q$ & $f$ & $f$ & 0 \\
$V$ & $a d j$ & 1 & 1 \\
\hline$q$ & & $T_{A}$ & 0 \\
\hline
\end{tabular}

Table 3. Matter content of two descriptions of SYM theory with $G_{c}=\mathrm{SU}(2)$ and 4 quarks.

like that only by its origin being a supersymmetric index for a nonconformal theory on the $S^{3} \times S^{1}$ manifold [8].

Classical SU(4) flavor symmetry group gets broken to $S P(4)$ [32] due to the modified quantum mechanical constraint. So, the true flavor symmetry group is $S P(4)$. Apart from the chiral symmetry breaking occurring for $N_{f}=2$ flavors it happens that the original electric theory can be described at low energies in terms of the free fields determined by gauge invariant operators. One has here the confinement with the chiral symmetry breaking which differs from the $s$-confinement $[37,38]$ studied from the SCI technique point of view in $[1,12]$. The matter content of both electric and magnetic theories is described in table 3 , where we put the matter content of both electric and magnetic theories in two subtables one atop of another. The upper one gives the matter content of the electric theory while the lower one reproduces the confining magnetic theory (which does not have the gauge fields).

Let us comment on what we have found so far. At the quantum level the original theory with SU(4) flavor symmetry is not complete at the arbitrary point in moduli space due to the quantum mechanical constraint. In this case SCI is equal to zero which is described by the relation $I_{E}=I_{M}=0$ for generic values of the fugacities $s_{i}$. The points of moduli space where the chiral symmetry breaking occurs bring the proper quantum gauge theory with its confining phase described by the dual theory of free chiral superfields. They are related to the special fugacity values for which SCIs diverge instead of vanishing. Based on this property we conclude that the equality $I_{E}=I_{M}$ describes the chiral symmetry breaking phenomenon for supersymmetric quantum chromodynamics theory with $N_{f}=2$ flavors. It should be stressed that although the formal expression for SCI of the electric theory (3.1) is built using the general prescription for constructing SCIs, the integration measure should be chosen in a rather careful way (one cannot use the contour $\mathbb{T}$ in (3.1)). Moreover, for the magnetic theory (3.2) even the formal expression of SCI cannot be derived using this prescription due to the appearance of the delta functions. A naive application of the general prescription in this case would produce infinity for the magnetic SCI, which is easily seen from the character of the absolutely antisymmetric tensor representation $T_{A}$ of $S P(4)$ :

$$
\chi_{T_{A}, S P(4)}=s_{1} s_{2}+s_{1} s_{2}^{-1}+s_{1}^{-1} s_{2}+s_{1}^{-1} s_{2}^{-1}+1 .
$$

The constant 1 entering this expression formally produces the diverging factor $\Gamma(1 ; p, q)$ from the plethystic exponential (which means that the sum in the exponential diverges). Very formally one can interpret $\Gamma(1 ; p, q)$ as the value of one of the delta functions in (3.2) when its argument vanishes. Consider again the case when $\phi_{1}+\phi_{3}=0$ implying $\phi_{2}+\phi_{4}=0$, which lead to $s_{1} s_{3}=s_{2} s_{4}=1$. Then in the expression (3.3) one easily recognizes the 
contribution from the character of fundamental representation of $S P(4)$

$$
\chi_{f, S P(4)}=s_{1}+s_{1}^{-1}+s_{2}+s_{2}^{-1},
$$

and in the expression (3.4) one sees only a part of the character of the $T_{A}$-representation (3.7).

In $[14,15]$ we already faced the fact that the prescription of computing SCIs in the form given in $[1,7]$ requires modifications in some particular cases. Here we also see that it does not cover theories with the quantum deformed moduli space. It would be nice to understand how this difficulty emerges from the localization procedure used for computing SCIs, which we do not discuss here.

\section{$4 \mathrm{SU}(N), N>2$, gauge group case}

Consider now the general $N$ case. According to [32] there are two different ways of getting the confinement with chiral symmetry breaking for $N>2$. At the classical level one has the following constraint

$$
\operatorname{det} M-B \widetilde{B}=0,
$$

where mesons $M$ and baryons $B, \widetilde{B}$ are defined as

$$
\begin{aligned}
M_{\widetilde{i}}^{i} & =Q^{i} \widetilde{Q}_{\tilde{i}}, \quad i, \widetilde{i}=1, \ldots, N, \\
B & =\frac{1}{N !} \epsilon_{i_{1} \ldots i_{N}} Q^{i_{1}} \ldots Q^{i_{N}}, \\
\widetilde{B} & =\frac{1}{N !} \epsilon_{\tilde{i}_{1} \ldots \widetilde{i}_{N}} \widetilde{Q}^{\widetilde{i}_{1}} \ldots \widetilde{Q}^{\widetilde{i}_{N}}
\end{aligned}
$$

In [32] it was shown that the classical constraint (4.1) is deformed quantum mechanically due to the one instanton effects to

$$
\operatorname{det} M-B \widetilde{B}=\Lambda^{2 N},
$$

where $\Lambda$ is some scale. Again, one has broken conformal symmetry and our index requires an appropriate interpretation in the context of non-conformal theories.

\subsection{Breaking to the diagonal subgroup: $\mathrm{SU}(N)_{l} \times \mathrm{SU}(N)_{r} \rightarrow \mathrm{SU}(N)_{d}$}

Condition (4.3) can be resolved by fixing

$$
B=\widetilde{B}=0, \quad M_{\widetilde{i}}^{i}=\Lambda^{2} \delta_{\tilde{i}}^{i}, \quad i, \widetilde{i}=1, \ldots, N,
$$

which leads to breaking of the flavor symmetry $\mathrm{SU}(N)_{l} \times \mathrm{SU}(N)_{r}$ to the diagonal subgroup $\mathrm{SU}(N)_{d}$. As a result one has the dual theories presented in table 4.

The electric theory SCI has the form

$$
I_{E}=\kappa_{N} \int_{\mathbb{T}_{d}^{N-1}} \frac{\prod_{i, j=1}^{N} \Gamma\left(u e^{2 \pi \mathrm{i} \theta_{i}} z_{j}, u^{-1} e^{-2 \pi \mathrm{i} \chi_{i}} z_{j}^{-1} ; p, q\right)}{\prod_{1 \leq i<j \leq N} \Gamma\left(z_{i} z_{j}^{-1}, z_{i}^{-1} z_{j} ; p, q\right)} \prod_{j=1}^{N-1} \frac{d z_{j}}{2 \pi \mathrm{i} z_{j}},
$$




\begin{tabular}{|c|c|c|c|c|}
\hline & $\mathrm{SU}(N)$ & $\mathrm{SU}(N)_{d}$ & $\mathrm{U}(1)_{B}$ & $\mathrm{U}(1)_{R}$ \\
\hline$Q$ & $f$ & $f$ & 1 & 0 \\
$\widetilde{Q}$ & $\bar{f}$ & $\bar{f}$ & -1 & 0 \\
$V$ & $a d j$ & 1 & 0 & 1 \\
\hline$M$ & & $a d j$ & 0 & 0 \\
\hline$S_{1}$ & & 1 & $N$ & 0 \\
\hline$S_{2}$ & & 1 & $-N$ & 0 \\
\hline
\end{tabular}

Table 4. Matter content of two descriptions of SYM theory with $G=\mathrm{SU}(N)$ and $2 N$ quarks with the symmetry breaking $\mathrm{SU}(N)_{l} \times \mathrm{SU}(N)_{r} \rightarrow \mathrm{SU}(N)_{d}$.

while the magnetic SCI is

$$
I_{M}=\frac{\Gamma\left(u^{ \pm N} ; p, q\right)}{(p ; p)_{\infty}^{N-1}(q ; q)_{\infty}^{N-1}} \prod_{1 \leq i<j \leq N} \Gamma\left(e^{ \pm 2 \pi \mathrm{i}\left(\theta_{i}-\theta_{j}\right)} ; p, q\right) \sum_{\tilde{\theta}_{j}} \prod_{i=1}^{N-1} \delta\left(\chi_{i}-\tilde{\theta}_{i}\right)
$$

where the sum goes over permutations of parameters $\left(\tilde{\theta}_{1}, \ldots, \tilde{\theta}_{N}\right)=\mathcal{P}\left(\theta_{1}, \ldots, \theta_{N}\right)$.

The equality $I_{E}=I_{M}$ is proved in the following section. Here we use parametrization of fugacities in the exponential form and

$$
\prod_{i=1}^{N} z_{i}=1, \quad \sum_{i=1}^{N} \theta_{i}=\sum_{i=1}^{N} \chi_{i}=0 .
$$

For $\theta_{i}=\chi_{i}$ one can easily recognize in $I_{E}$ contributions of the characters of respective electric theory field representations as described in table 4. As to the magnetic theory, the meson field $M$ described by the adjoint representation and respective character

$$
\chi_{a d j, \mathrm{SU}(N)}=\sum_{i, j=1}^{N} e^{2 \pi \mathrm{i}\left(\theta_{i}-\theta_{j}\right)}-1,
$$

yields the $\theta_{j}$-dependent term in (4.6) multiplied by the diverging factor $\Gamma(1 ; p, q)^{N-1}$, which formally plays the role of the product of delta functions. The contribution to $I_{M}$ of the scalar fields $S_{1}$ and $S_{2}$ is described by the terms $\Gamma\left(u^{ \pm N} ; p, q\right)$ using the standard prescription. So, we see that the original recipe of building SCIs for $\mathrm{SU}(N)$ supersymmetric field theories with chiral symmetry breaking requires appropriate modification on both electric (namely, by correct choice of the integration contour $\mathbb{T}_{d}$ ) and magnetic (by correct description of singularities in the distributional sense) sides.

We would like to note that for $N=4$, i.e. for $\mathrm{SU}(4)$ gauge theory there are several dual theories as described in [13]. This means that a similar chiral symmetry breaking should take place in three other interacting $\mathrm{SU}(4)$-gauge field theories with $N_{f}=4$. We have checked that the expression for SCIs (4.6) is invariant with respect to transformations of elliptic hypergeometric integrals indicated in [13], i.e. the latter theories have the same index. It would be interesting to investigate moduli spaces and other physical properties 


\begin{tabular}{|c|c|c|c|c|}
\hline & $\mathrm{SU}(N)$ & $\mathrm{SU}(N)_{l}$ & $\mathrm{SU}(N)_{r}$ & $\mathrm{U}(1)_{R}$ \\
\hline$Q$ & $f$ & $f$ & 1 & 0 \\
$\widetilde{Q}$ & $\bar{f}$ & 1 & $\bar{f}$ & 0 \\
$V$ & $a d j$ & 1 & 1 & 1 \\
\hline$M$ & & $f$ & $\bar{f}$ & 0 \\
\hline$S$ & & 1 & 1 & 0 \\
\hline
\end{tabular}

Table 5. Matter content of two descriptions of SYM theory with $G_{c}=\mathrm{SU}(N)$ and $2 N$ quarks with broken $\mathrm{U}(1)_{B}$.

of these multiple dual theories to see how the chiral symmetry breaking arises in them. The general physical properties of these and other more general dualities lying outside the conformal windows described in [13] are not investigated appropriately yet. As a correction, we mention that vanishing of SCIs stated in [13] is wrong in general - there are singularities describing interesting physics and all corresponding SCIs should be reconsidered from this point of view.

\subsection{Breaking of $\mathrm{U}(1)_{B}$}

The constraint (4.3) can be resolved also by fixing

$$
B=-\widetilde{B}=\Lambda^{N}, \quad M_{\tilde{i}}^{i}=0, \quad i, \widetilde{i}=1, \ldots, N,
$$

which leads to breaking of the $\mathrm{U}(1)_{B}$-symmetry. As a result in the infrared fixed point one gets the dual field description as described in table 5 .

The electric SCI has the same form

$$
I_{E}=\kappa_{N} \int_{\mathbb{T}_{d}^{N-1}} \frac{\prod_{i, j=1}^{N} \Gamma\left(x_{i} u z_{j}, y_{i}^{-1} u^{-1} z_{j}^{-1} ; p, q\right)}{\prod_{1 \leq i<j \leq N} \Gamma\left(z_{i} z_{j}^{-1}, z_{i}^{-1} z_{j} ; p, q\right)} \prod_{j=1}^{N-1} \frac{d z_{j}}{2 \pi \mathrm{i} z_{j}},
$$

with $\prod_{i=1}^{N} z_{i}=1, \prod_{i=1}^{N} x_{i}=\prod_{i=1}^{N} y_{i}=1$, while the magnetic index has a different form

$$
I_{M}=\prod_{i, j=1}^{N} \Gamma\left(x_{i} y_{j}^{-1} ; p, q\right) \frac{\delta(N \varphi)}{(p ; p)_{\infty}(q ; q)_{\infty}},
$$

where $\varphi$ is a real variable appearing from the exponential parametrization of the $\mathrm{U}(1)_{B^{-}}$ fugacity, $u=e^{2 \pi i \varphi}$. The equality $I_{E}=I_{M}$ in this case is also proven in the next section. Again, for discrete values of the fugacity $u, u^{N}=1$, the electric index uses a nontrivial modification of the integration contour $\mathbb{T}_{d}$ with clear contribution from characters of the fundamental ( $Q$-field) and antifundamental ( $\widetilde{Q}$-field) representations. In the magnetic case the situation is trickier. The tensor meson field $M$ character yields the $x_{i}, y_{i}$-dependent part of the expression (4.9) whereas the delta function $\delta(N \varphi)$ is modelled by the character of the scalar field $S$ which has zero charges with respect to all groups and so yields $\Gamma(t ; p, q)$ at $t=1$ which plays the role of the delta function, i.e. a modification of the original recipe of building SCIs is needed in this case as well. 
Again, for $N=4$ similar chiral symmetry breaking should take place in three other SU(4)-gauge group dual theories with $N_{f}=4$ described in [13]. We have checked that the expression for magnetic SCI (4.9) is invariant with respect to transformations of EHIs given in [13], i.e. these theories have the same index.

\section{Proofs}

\section{$5.1 \quad N_{f}=N=2$ case}

Let us prove relations for SCIs presented in the previous section. Consider first the chiral symmetry breaking in $\mathcal{N}=1 \mathrm{SYM}$ theory with $\mathrm{SU}(2)$ gauge group and $N_{f}=2$ flavors. Properties of SCIs in this particular case were discussed in $[29,36]$, but here we would like to give an independent consideration.

Take the $s$-confining theory with the same gauge group $\mathrm{SU}(2)$ and $N_{f}=3$ flavors studied in [32]. Note that all $s$-confining theories were thought to be classified in [37, 38], however other examples of such theories were discovered in [12] using the SCI technique. Since for $G_{c}=\mathrm{SU}(2)$ the fundamental and antifundamental representations are equivalent, the flavor group extends to SU(6) and quark fields unify to its fundamental representation. Denoting $t_{1,2,3}^{-1}=s_{4,5,6}$ in $(2.5)$ we come to the electric SCI

$$
I_{E}=\frac{(p ; p)_{\infty}(q ; q)_{\infty}}{2} \int_{\mathbb{T}} \frac{\prod_{j=1}^{6} \Gamma\left(s_{j} z^{ \pm 1} ; p, q\right)}{\Gamma\left(z^{ \pm 2} ; p, q\right)} \frac{d z}{2 \pi \mathrm{i} z}
$$

where $\left|s_{j}\right|<1$ and $\prod_{j=1}^{6} s_{j}=p q$. This integral is known as the elliptic beta integral and its evaluation [2] yields the magnetic SCI (cf. with (2.8))

$$
I_{M}=\prod_{1 \leq j<k \leq 6} \Gamma\left(s_{j} s_{k} ; p, q\right)
$$

It is possible to reduce the equality $I_{E}=I_{M}$ to $N_{f}=2$ case by taking the limit

$$
s_{5} s_{6}=p q e^{\epsilon}, \quad \epsilon \rightarrow 0 .
$$

Indeed, from the inversion relation for $\Gamma(z ; p, q)$ one has $\Gamma\left(s_{5} z, s_{6} z^{-1} ; p, q\right) \rightarrow 1$ and integral (5.1) simplifies to (3.1), where the integration contour should be inevitably deformed due to the balancing condition $s_{1} s_{2} s_{3} s_{4}=1$ emerging for $\epsilon=0$.

Let $\epsilon$ be a small positive number. Denote

$$
s_{1}=\alpha w, \quad s_{2}=\alpha w^{-1}, \quad s_{3}=\beta y, \quad s_{4}=\beta y^{-1} .
$$

Then

$$
I_{M}=\Gamma\left(s_{5} s_{6}, \alpha^{2}, \beta^{2}, \alpha \beta w^{ \pm 1} y^{ \pm 1} ; p, q\right) \prod_{m=5}^{6} \Gamma\left(\alpha s_{m} w^{ \pm 1}, \beta s_{m} y^{ \pm 1} ; p, q\right)
$$

Because

$$
\Gamma\left(s_{5} s_{6} ; p, q\right) \underset{\epsilon \rightarrow 0}{=} \epsilon(p ; p)_{\infty}(q ; q)_{\infty}+\mathrm{O}\left(\epsilon^{2}\right)
$$


the integral $I_{E}$ (5.1) is proportional to $\epsilon$ and, for generic values of other parameters, it vanishes for $\epsilon=0$. However, for special values of $\alpha, \beta, w$, and $y$ one has poles in (5.5) and corresponding singularities may alter the integral value. Let us consider the situation for singular points $w=y$ (corresponding to $\left.s_{1} s_{4}=1\right), w=y^{-1}$ (corresponding to $s_{1} s_{3}=1$ ), and $\alpha^{2}=1$ (corresponding to $s_{1} s_{2}=1$ ).

The balancing condition $s_{5} s_{6} \alpha^{2} \beta^{2}=p q$ can be written in the form

$$
\alpha \beta=e^{-\epsilon / 2}
$$

Actually, on the right hand side of (5.7) one may have the minus sign, but it can be removed by the change $y \rightarrow-y$ in the original notation and therefore we stick to the positive sign in (5.7). Keeping $\epsilon>0$ one has $|\alpha \beta|<1$, i.e. it is possible to choose $|\alpha|,|\beta|<1$. Suppose that $w, y \in \mathbb{T}$, i.e. $|w|,|y|=1$. Then all parameters of the elliptic beta integral are of modulus less than 1 and its evaluation (5.5) holds true.

To see the nature of singularities emerging at $y=w^{ \pm 1}$ let us assume that $\alpha^{2} \neq 1$, multiply $I_{E}$ by a function $f(y)$ holomorphic near the unit circle and integrate over the variable $y$ :

$$
\begin{aligned}
\int_{\mathbb{T}} f(y) I_{E} \frac{d y}{2 \pi \mathrm{i} y}=\Gamma\left(s_{5} s_{6}, \alpha^{2}, \beta^{2} ; p, q\right) \prod_{m=5}^{6} \Gamma\left(\alpha s_{m} w^{ \pm 1} ; p, q\right) \\
\quad \times \int_{\mathbb{T}} f(y) \Gamma\left(\alpha \beta w^{ \pm 1} y^{ \pm 1} ; p, q\right) \prod_{m=5}^{6} \Gamma\left(\beta s_{m} y^{ \pm 1} ; p, q\right) \frac{d y}{2 \pi \mathrm{i} y} .
\end{aligned}
$$

Since we may keep absolute values of $s_{5}$ and $s_{6}$ sufficiently small, so that $\left|\beta s_{5,6}\right|<1$, there are no problems with the integration of $s_{5,6}$-dependent elliptic gamma functions in (5.8). The term $\Gamma\left(\alpha \beta w^{ \pm 1} y^{ \pm 1} ; p, q\right)$ has the following sequences of poles and zeros

- poles: $\quad y_{\text {in }}=\alpha \beta w^{ \pm 1} p^{i} q^{j}, \quad y_{\text {out }}=\frac{1}{\alpha \beta} w^{ \pm 1} p^{-i} q^{-j}$

- zeros: $y=\frac{1}{\alpha \beta} w^{ \pm 1} p^{i+1} q^{j+1}, \alpha \beta w^{ \pm 1} p^{-i-1} q^{-j-1}$,

where $i, j \in \mathbb{Z}_{\geq 0}$ and the in-poles converge to zero $y=0$ and out-poles go to infinity. Because of the taken constraints the unit circle separates in and out poles for $\epsilon>0$. However, for $\epsilon \rightarrow 0$ one has $\alpha \beta \rightarrow 1$ and two pairs of poles at $\alpha \beta w^{ \pm 1}$ and $w^{ \pm 1} / \alpha \beta$ start to pinch $\mathbb{T}$. Therefore we deform the contour of integration $\mathbb{T}$ to $\mathcal{C}$ such that only the residues of the $\alpha \beta w^{ \pm 1}$-poles are picked up during this deformation and there are no singularities lying on $\mathcal{C}$. These distinguished poles are simple for $w^{2} \neq 1$ (i.e., $s_{1} \neq s_{2}$ ), which is assumed in the following. As a result we obtain

$$
\begin{aligned}
& \int_{\mathbb{T}} f(y) I_{E} \frac{d y}{2 \pi \mathrm{i} y}=\Gamma\left(s_{5} s_{6}, \alpha^{2}, \beta^{2} ; p, q\right) \prod_{m=5}^{6} \Gamma\left(\alpha s_{m} w^{ \pm 1} ; p, q\right) \\
& \quad \times\left(\frac{f(\alpha \beta w)}{(p ; p)_{\infty}(q ; q)_{\infty}} \Gamma\left((\alpha \beta)^{2},(\alpha \beta w)^{2}, w^{-2} ; p, q\right) \prod_{m=5}^{6} \Gamma\left(\beta s_{m} \alpha \beta w, \frac{s_{m}}{\alpha w} ; p, q\right)\right.
\end{aligned}
$$




$$
\begin{aligned}
& +\frac{f\left(\alpha \beta w^{-1}\right)}{(p ; p)_{\infty}(q ; q)_{\infty}} \Gamma\left((\alpha \beta)^{2},\left(\alpha \beta w^{-1}\right)^{2}, w^{2} ; p, q\right) \prod_{m=5}^{6} \Gamma\left(\beta s_{m} \alpha \beta w^{-1}, \frac{s_{m} w}{\alpha} ; p, q\right) \\
& \left.+\int_{\mathcal{C}} f(y) \Gamma\left(\alpha \beta w^{ \pm 1} y^{ \pm 1} ; p, q\right) \prod_{m=5}^{6} \Gamma\left(\beta s_{m} y^{ \pm 1} ; p, q\right) \frac{d y}{2 \pi \mathrm{i} y}\right)
\end{aligned}
$$

where we used the relation

$$
\lim _{x \rightarrow 1}(1-x) \Gamma(x ; p, q)=\frac{1}{(p ; p)_{\infty}(q ; q)_{\infty}} .
$$

The residue factors $\Gamma\left((\alpha \beta)^{2} ; p, q\right)$ diverge for $\epsilon \rightarrow 0$, but $\Gamma\left(s_{5} s_{6},(\alpha \beta)^{2} ; p, q\right)=1$ and we obtain the finite product. However, the integral over $\mathcal{C}$ remains finite and its product with $\Gamma\left(s_{5} s_{6} ; p, q\right)$ vanishes. As a result, for $\epsilon=0$ we obtain

$$
\int_{\mathbb{T}} f(y) I_{E} \frac{d y}{2 \pi \mathrm{i} y}=\frac{\Gamma\left(\alpha^{ \pm 2}, w^{ \pm 2} ; p, q\right)}{(p ; p)_{\infty}(q ; q)_{\infty}}\left(f(w)+f\left(w^{-1}\right) .\right.
$$

Denote $y=e^{2 \pi \mathrm{i} \theta}, w=e^{2 \pi \mathrm{i} \chi}$ and pass to the integration over real variable $\theta$. Because of the arbitrariness of the function $f(y)$, we can give to the function $I_{E}$ a distributional sense and write

$$
\begin{gathered}
\frac{(p ; p)_{\infty}(q ; q)_{\infty}}{2} \int_{\mathbb{T}_{d}} \frac{\Gamma\left(\alpha w^{ \pm 1} z^{ \pm 1}, \alpha^{-1} y^{ \pm 1} z^{ \pm 1} ; p, q\right)}{\Gamma\left(z^{ \pm 2} ; p, q\right)} \frac{d z}{2 \pi \mathrm{i} z} \\
=\frac{\Gamma\left(\alpha^{ \pm 2}, w^{ \pm 2} ; p, q\right)}{(p ; p)_{\infty}(q ; q)_{\infty}}(\delta(\theta+\chi)+\delta(\theta-\chi)),
\end{gathered}
$$

where $\delta(\theta)$ is the 1-periodic Dirac delta function. Note that the limit $\epsilon \rightarrow 0$ inevitably enforces the change of the integration contour in $I_{E}$ from $\mathbb{T}$ to $\mathbb{T}_{d}$, which is a deformation of $\mathbb{T}$ such that the sequences of poles $\alpha w^{ \pm 1} p^{i} q^{j}$ and $\alpha^{-1} y^{ \pm 1} p^{i} q^{j}$ with $i, j \in \mathbb{Z}_{\geq 0}$ lie inside $\mathbb{T}_{d}$ and their reciprocals - outside of this contour. If $|\alpha|<1$ then some poles from the second set lie outside $\mathbb{T}$, i.e. the contour deformation is not infinitesimal. For symmetric functions $f(z)=f\left(z^{-1}\right)$, the equality (5.10) has an interpretation as the inversion relation for an integral operator introduced in [39], which was demonstrated in [40]. In turn, it was identified in [41] with one of the Coxeter relations for permutation groups.

Consider now the singularities of $I_{E}$ at $\alpha^{2}=1$. For that we multiply $I_{E}$ by a holomorphic function $f(\alpha)$ and integrate over $\alpha$ along the contour $\mathcal{C}$ which is a deformation of $\mathbb{T}$ near the points $\alpha= \pm 1$ such that it passes in between the points $\alpha=1$ and $\alpha=e^{-\epsilon / 2}$ on the one side and points $\alpha=-1$ and $\alpha=-e^{-\epsilon / 2}$ on the other side. Again, in the limit $\epsilon \rightarrow 0$ two pairs of poles pinch the integration contour and we deform $\mathcal{C}$ to an infinitesimal deformation of $\mathbb{T}$ such that both points $\alpha= \pm 1$ lie inside it, and pick up $\alpha= \pm 1$ pole residues. Repeating considerations similar to the previous case we obtain

$$
\begin{gathered}
\int_{\mathcal{C}} f(\alpha) I_{E} \frac{d \alpha}{2 \pi \mathrm{i} \alpha}=\Gamma\left(s_{5} s_{6}, e^{-\epsilon / 2} w^{ \pm 1} y^{ \pm 1} ; p, q\right) \int_{\mathcal{C}} f(\alpha) \Gamma\left(\alpha^{2}, e^{-\epsilon} \alpha^{-2} ; p, q\right) \\
\times \prod_{m=5}^{6} \Gamma\left(\alpha s_{m} w^{ \pm 1}, e^{-\epsilon / 2} \alpha^{-1} s_{m} y^{ \pm 1} ; p, q\right) \frac{d \alpha}{2 \pi \mathrm{i} \alpha}
\end{gathered}
$$




$$
\underset{\epsilon \rightarrow 0}{=} \frac{\Gamma\left(w^{ \pm 1} y^{ \pm 1} ; p, q\right)}{(p ; p)_{\infty}(q ; q)_{\infty}} \frac{f(1)+f(-1)}{2}
$$

Denote $\alpha=e^{2 \pi i \varphi}$ and pass to the integration over the real variable $\varphi \in[0,1[$. Then we can write in the distributional sense

$$
\begin{aligned}
& \frac{(p ; p)_{\infty}(q ; q)_{\infty}}{2} \int_{\mathbb{T}_{d}} \frac{\Gamma\left(\alpha w^{ \pm 1} z^{ \pm 1}, \alpha^{-1} y^{ \pm 1} z^{ \pm 1} ; p, q\right)}{\Gamma\left(z^{ \pm 2} ; p, q\right)} \frac{d z}{2 \pi \mathrm{i} z} \\
& =\frac{\Gamma\left(w^{ \pm 1} y^{ \pm 1} ; p, q\right)}{(p ; p)_{\infty}(q ; q)_{\infty}} \frac{\delta(\varphi)+\delta(\varphi-1 / 2)}{2} .
\end{aligned}
$$

Equivalently we can write $\delta(\varphi)+\delta(\varphi-1 / 2)=2 \delta(2 \varphi)$, because of the periodicity of the delta function. For these considerations to be valid we have to assume that $y^{ \pm 1} w^{ \pm 1} \neq 1$, i.e. the previously considered regime of parameters and the current one should not overlap, which we assumed.

Return now from notation (5.4) to the original one $s_{i}=e^{2 \pi i \phi_{i}}$, which means that $\phi_{1}=\varphi+\theta, \phi_{2}=\varphi-\theta, \phi_{3}=-\varphi+\chi, \phi_{4}=-\varphi-\chi$. Then the arguments of our delta functions are $2 \varphi=\phi_{1}+\phi_{2}, \theta+\chi=\phi_{1}+\phi_{3}$, and $\theta-\chi=\phi_{1}+\phi_{4}$. Therefore summing righthand sides of (5.10) and (5.12) we come to the expression for magnetic SCI (3.2) which we wanted to prove. Note that due to our constraints on the parameters the supports of three delta functions do not overlap.

\subsection{The higher rank case, $N_{f}=N>2$}

Consider the general case $N_{f}=N>2$. The situation with breaking $\mathrm{SU}\left(N_{f}\right)_{l} \times \mathrm{SU}\left(N_{f}\right)_{r} \times$ $\mathrm{U}(1)_{B} \rightarrow \mathrm{SU}\left(N_{f}\right)_{d} \times \mathrm{U}(1)_{B}$ is similar to the one described above for $y=w^{ \pm 1}$. Analysis of the $\mathrm{U}(1)_{B}$-breaking, $\mathrm{SU}\left(N_{f}\right)_{l} \times \mathrm{SU}\left(N_{f}\right)_{r} \times \mathrm{U}(1)_{B} \rightarrow \mathrm{SU}\left(N_{f}\right)_{l} \times \mathrm{SU}\left(N_{f}\right)_{r}$, is analogous to the investigation of $\alpha^{2}=1$ singularities above.

We start from the $s$-confining theory with $\mathrm{SU}(N)$ gauge group with $N_{f}=N+1$ flavors. The electric theory SCI is

$$
I_{E}=\kappa_{N} \int_{\mathbb{T}^{N-1}} \frac{\prod_{i=1}^{N+1} \prod_{j=1}^{N} \Gamma\left((p q)^{\frac{1}{2(N+1)}} x_{i} v z_{j},(p q)^{\frac{1}{2(N+1)}} y_{i}^{-1} v^{-1} z_{j}^{-1} ; p, q\right)}{\prod_{1 \leq i<j \leq N} \Gamma\left(z_{i} z_{j}^{-1}, z_{i}^{-1} z_{j} ; p, q\right)} \prod_{j=1}^{N-1} \frac{d z_{j}}{2 \pi \mathrm{i} z_{j}}
$$

where $\prod_{j=1}^{N+1} x_{j}=\prod_{j=1}^{N+1} y_{j}=1$, so that the balancing condition is satisfied automatically. It admits exact evaluation yielding the magnetic theory SCI

$$
I_{M}=\prod_{i=1}^{N+1} \Gamma\left((p q)^{\frac{N}{2(N+1)}} x_{i}^{-1} v^{N},(p q)^{\frac{N}{2(N+1)}} y_{i} v^{-N} ; p, q\right) \prod_{i, j=1}^{N+1} \Gamma\left((p q)^{\frac{1}{N+1}} x_{i} y_{j}^{-1} ; p, q\right) .
$$

As in the previous considerations we would like to integrate out one flavor by taking the limit $(p q)^{\frac{1}{N+1}} x_{N+1} y_{N+1}^{-1}=p q e^{\epsilon}, \epsilon \rightarrow 0$. Introduce new variables $a_{i}, b_{i}$, and $u$ :

$$
x_{i}=\frac{a_{i}}{x_{N+1}^{1 / N}}, \quad y_{i}=\frac{b_{i}}{y_{N+1}^{1 / N}}, \quad i=1, \ldots, N, \quad v=(p q)^{-\frac{1}{2(N+1)}} x_{N+1}^{1 / N} u,
$$


which will play the role of fugacities for $N_{f}=N$ reduced theory, $\prod_{i=1}^{N} a_{i}=\prod_{i=1}^{N} b_{i}=1$. Then the indices take the form

$$
\begin{aligned}
I_{E}= & \kappa_{N} \int_{\mathbb{T}^{N-1}} \frac{\prod_{i, j=1}^{N} \Gamma\left(a_{i} u z_{j}, e^{-\epsilon / N} b_{i}^{-1} u^{-1} z_{j}^{-1} ; p, q\right)}{\prod_{1 \leq i<j \leq N} \Gamma\left(z_{i} z_{j}^{-1}, z_{i}^{-1} z_{j} ; p, q\right)} \\
& \times \prod_{j=1}^{N} \Gamma\left(x_{N+1}^{\frac{N+1}{N}} u z_{j}, y_{N+1}^{-\frac{N+1}{N}} u^{-1} z_{j}^{-1} e^{-\epsilon / N} ; p, q\right) \prod_{j=1}^{N-1} \frac{d z_{j}}{2 \pi \mathrm{i} z_{j}}
\end{aligned}
$$

and

$$
\begin{aligned}
I_{M}= & \Gamma\left(p q e^{\epsilon}, u^{N}, e^{-\epsilon} u^{-N} ; p, q\right) \prod_{i, j=1}^{N} \Gamma\left(e^{-\epsilon / N} a_{i} b_{j}^{-1} ; p, q\right) \\
& \times \prod_{i=1}^{N} \Gamma\left(x_{N+1}^{\frac{N+1}{N}} a_{i}^{-1} u^{N}, e^{-\epsilon} y_{N+1}^{-\frac{N+1}{N}} b_{i} u^{-N} ; p, q\right) \\
& \times \prod_{i=1}^{N} \Gamma\left(p q e^{\epsilon} y_{N+1}^{\frac{N+1}{N}} b_{i}^{-1}, p q e^{\epsilon} x^{-\frac{N+1}{N}} a_{i} ; p, q\right) .
\end{aligned}
$$

In the limit $\epsilon \rightarrow 0$ this expression vanishes for generic values of the parameters. The singular manifold of fugacities requiring special consideration is determined by the poles of elliptic gamma functions in (5.16). The fugacity $x_{N+1}$ (or $y_{N+1}$ ) is an arbitrary variable and we can keep corresponding poles away from $\mathbb{T}$. Therefore for fugacities $a_{i}, b_{i}$, and $u$ near the unit circle the only singular points of interest are $a_{i}=b_{j}$ and $u^{N}=1$. In order to see the structure of singularities in the first case, we multiply $I_{E}$ (or $I_{M}$ ) by a holomorphic function $f\left(b_{1}, \ldots, b_{N}\right)$ and integrate over the variables $b_{1}, \ldots, b_{N-1} \in \mathbb{T}$. The multipliers $\Gamma\left(e^{-\epsilon / N} a_{i} b_{j}^{-1} ; p, q\right)$ have the poles

$$
\text { in }: \quad b_{j}=e^{-\epsilon / N} a_{i}, \quad i=1, \ldots, N, j=1, \ldots, N-1,
$$

lying inside $\mathbb{T}$, and

$$
\text { out : } \quad b_{N}^{-1}=b_{1} \ldots b_{N-1}=e^{\epsilon / N} a_{i}^{-1}, \quad i=1, \ldots, N,
$$

lying outside $\mathbb{T}$ for any particular $b_{k}$. Since $a_{i} \in \mathbb{T}$, for $\epsilon \rightarrow 0$ all in poles approach $\mathbb{T}$ from inside. Positions of the out poles depend on the order of integration in $b_{i}$ and their values. Suppose we integrate first over $b_{1}$, then $b_{2}$, etc. Then for the pole $b_{1}=e^{\epsilon / N} / a_{j} b_{2} \ldots b_{N-1}$ there exist such values of $b_{2}, \ldots, b_{N-1} \in \mathbb{T}$ that $b_{1}=e^{\epsilon / N} a_{k}$ for $k=1, \ldots, N$ and for $\epsilon \rightarrow 0$ we have pinching of the integration contour near the points $b_{1}=a_{k}$. To escape such a pinching we shrink the integration contours a little to pick up the residues of the $b_{i}=e^{-\epsilon / N} a_{i}$ poles lying inside $\mathbb{T}$ (like in the $N=2$ case). After taking sequentially $N-1$ "residues of residues" in integration variables, say at the point $b_{i}=a_{i}$, on the last step we obtain the term

$$
\Gamma\left(e^{-\epsilon / N} a_{N} b_{N}^{-1} ; p, q\right)=\Gamma\left(e^{-\epsilon} ; p, q\right),
$$

which diverges and, being multiplied by $\Gamma\left(p q e^{\epsilon} ; p, q\right)$, yields the finite answer. Evidently, we can take residues in arbitrary possible order $b_{i}=e^{-\epsilon / N} a_{j}, j=1, \ldots, N$, each of which 
yields different final result. Only the highest order residues survive in the limit $\epsilon \rightarrow 0$ since all lower order residues vanish due to the multiplier $\Gamma\left(p q e^{\epsilon} ; p, q\right)$. As a result we obtain

$$
\begin{gathered}
\int_{\mathbb{T}^{N-1}} f\left(b_{1}, \ldots, b_{N}\right) I_{M} \prod_{j=1}^{N-1} \frac{d b_{j}}{2 \pi \mathrm{i} b_{j}} \underset{\epsilon \rightarrow 0}{=} \frac{\Gamma\left(u^{ \pm N} ; p, q\right)}{(p ; p)_{\infty}^{N-1}(q ; q)_{\infty}^{N-1}} \\
\times \prod_{1 \leq i<j \leq N} \Gamma\left(a_{i} a_{j}^{-1}, a_{i}^{-1} a_{j} ; p, q\right) \sum_{\tilde{a}_{j}} f\left(\tilde{a}_{1}, \ldots, \tilde{a}_{N}\right),
\end{gathered}
$$

where summation goes over all permutations of parameters appearing from different orders of taking residues, $\left(\tilde{a}_{1}, \ldots, \tilde{a}_{N}\right)=\mathcal{P}\left(a_{1}, \ldots, a_{N}\right)$.

To tackle the singularities at $u^{N}=1$ we multiply $I_{M}$ by a holomorphic function $f(u)$ and integrate over $u$ along the contour which is an infinitesimal deformation of $\mathbb{T}$ passing in between the points $u=e^{2 \pi \mathrm{i} k / N}$ and $u=e^{-\epsilon / N} e^{2 \pi \mathrm{i} k / N}, k=0, \ldots, N-1$. Then we deform the integration contour and pick up the residues at $u=e^{2 \pi \mathrm{i} k / N}$. For $\omega^{N}=1$ one has

$$
\lim _{u \rightarrow \omega}(1-\omega / u) \Gamma\left(u^{N} ; p, q\right)=-\frac{1}{N(p ; p)_{\infty}(q ; q)_{\infty}} .
$$

The contours for computing residues are oriented clockwise, which results in the extra minus sign and yields

$$
\int_{\mathbb{T}_{d}} f(u) I_{M} \frac{d u}{2 \pi \mathrm{i} u}=\prod_{1 \leq i, j \leq N} \Gamma\left(a_{i} b_{j}^{-1} ; p, q\right) \frac{1}{N(p ; p)_{\infty}(q ; q)_{\infty}} \sum_{k=0}^{N-1} f\left(e^{2 \pi \mathrm{i} k / N}\right) .
$$

Introducing the angular variables $a_{i}=e^{2 \pi \mathrm{i} \theta_{i}}, b_{i}=e^{2 \pi \mathrm{i} \chi_{i}}$ and $u=e^{2 \pi \mathrm{i} \varphi}$, we can write in the distributional sense

$$
\begin{aligned}
\kappa_{N} \int_{\mathbb{T}_{d}^{N-1}} \frac{\prod_{i, j=1}^{N} \Gamma\left(e^{2 \pi \mathrm{i}\left(\theta_{i}+\varphi\right)} z_{j}, e^{-2 \pi \mathrm{i}\left(\chi_{i}+\varphi\right)} z_{j}^{-1} ; p, q\right)}{\prod_{1 \leq i<j \leq N} \Gamma\left(z_{i} z_{j}^{-1}, z_{i}^{-1} z_{j} ; p, q\right)} \prod_{j=1}^{N-1} \frac{d z_{j}}{2 \pi \mathrm{i} z_{j}} \\
=\frac{\Gamma\left(u^{ \pm N} ; p, q\right)}{(p ; p)_{\infty}^{N-1}(q ; q)_{\infty}^{N-1}} \prod_{1 \leq i<j \leq N} \Gamma\left(a_{i} a_{j}^{-1}, a_{i}^{-1} a_{j} ; p, q\right) \sum_{\tilde{\theta}_{j}} \prod_{k=1}^{N-1} \delta\left(\chi_{k}-\tilde{\theta}_{k}\right) \\
\quad+\prod_{1 \leq i, j \leq N} \Gamma\left(a_{i} b_{j}^{-1} ; p, q\right) \frac{\delta(N \varphi)}{(p ; p)_{\infty}(q ; q)_{\infty}}
\end{aligned}
$$

where the sum $\sum_{\tilde{\theta}_{j}}$ goes over all $N$ ! permutations of the variables $\left(\theta_{1}, \ldots, \theta_{N}\right)$ and $\delta(N \varphi)=$ $(1 / N) \sum_{k=0}^{N-1} \delta(\varphi-k / N)$. This is a general formula describing simultaneously both cases of chiral symmetry breaking.

Interestingly, for $N=4$ the expression (5.19) has an extended symmetry generated by the reflection of fugacities and multiplication by some elliptic gamma functions described in [13] in association with three more dual theories with nontrivial SU(4)-gauge group interaction. 


\subsection{The case $N_{f}<N$}

Take the electric theory with $G_{c}=\mathrm{SU}(2)$ and a single flavor $N_{f}=1$. Corresponding SCI has the form

$$
\frac{(p ; p)_{\infty}(q ; q)_{\infty}}{2} \int_{\mathcal{C}} \frac{\Gamma\left((p q)^{-\frac{1}{2}} e^{ \pm \mathrm{i} \theta} z^{ \pm 1} ; p, q\right)}{\Gamma\left(z^{ \pm 2} ; p, q\right)} \frac{d z}{2 \pi \mathrm{i} z},
$$

where the integration contour $\mathcal{C}$ separates the poles converging to zero from their reciprocals. It can be formally obtained from the $N_{f}=2$ index by setting $\alpha=\sqrt{p q}$. For generic values of $\theta$ this integral vanishes, as a consequence of the elliptic beta integral evaluation. However, it is not completely clear for which values of $\theta$ there are singularities allowing one to obtain a non-zero answer in the distributional sense. It is not legitimate to simply substitute $\alpha=\sqrt{p q}$ into (5.10) since that relation was obtained under the condition $\left|\beta s_{5}\right|,\left|\beta s_{6}\right|<1$. For $\alpha \rightarrow \sqrt{p q}$ one has $\beta^{2} \rightarrow(p q)^{-1}$, so that $s_{5} s_{6} \beta^{2} \rightarrow 1$ and there emerge additional pinchings of the $y$-variable integration contour.

Consider SCI for the pure SU(2) SYM theory, i.e. the $N_{f}=0$ case. This theory has $R$-symmetry anomaly and the corresponding SCI is described not by an EHI, but by a theta hypergeometric integral [3] (i.e. no balancing condition is present):

$$
I_{\text {pure }, \mathrm{SU}(2)}=\frac{(p ; p)_{\infty}(q ; q)_{\infty}}{2} \int_{\mathbb{T}} \frac{1}{\Gamma\left(z^{ \pm 2} ; p, q\right)} \frac{d z}{2 \pi \mathrm{i} z} .
$$

As was mentioned already, this formula emerges from the formal free field considerations. Actually, absence of the $R$-symmetry makes it questionable how to define the superconformal index on the $S^{3} \times S^{1}$ manifold. Therefore it is not completely clear what kind of data are described by the expression (5.21).

Nevertheless, the integral (5.21) can be evaluated explicitly. To compute it, we use the inversion formula for elliptic gamma functions

$$
\frac{1}{\Gamma\left(z^{ \pm 2} ; p, q\right)}=\theta\left(z^{2} ; p\right) \theta\left(z^{-2} ; q\right)
$$

where the theta function is defined as

$$
\theta(z ; p)=(z ; p)_{\infty}\left(p z^{-1} ; p\right)_{\infty}=\frac{1}{(p ; p)_{\infty}} \sum_{k \in \mathbb{Z}}(-1)^{k} p^{k(k-1) / 2} z^{k}
$$

Applying the latter series expansion for theta functions twice we get

$$
\begin{aligned}
I_{\text {pure SU(2) }} & =\frac{1}{2} \sum_{k, l \in \mathbb{Z}}(-1)^{k+l} p^{k(k-1) / 2} q^{l(l-1) / 2} \int_{\mathbb{T}} z^{2(k-l)} \frac{d z}{2 \pi \mathrm{i} z} \\
& =\frac{1}{2} \sum_{k \in \mathbb{Z}}(p q)^{k(k-1) / 2}=\frac{1}{2}(p q ; p q)_{\infty} \theta(-1 ; p q) .
\end{aligned}
$$

Using the plethistic exponential we can also write

$$
I_{\text {pure SU }(2)}=(p q ; p q)_{\infty}(-p q ; p q)_{\infty}^{2}=\exp \left(-\sum_{n=1}^{\infty} \frac{(p q)^{n}+2(-p q)^{n}}{n\left(1-(p q)^{n}\right)}\right) .
$$

The physical meaning of this relation is not completely clear. Perhaps, the right-hand side expression in (5.23) hints on the formation of the gaugino condensate [42, 43]. 


\begin{tabular}{|c|c|c|c|}
\hline & $S P(2 N)$ & $S P(2(N+1))$ & $\mathrm{U}(1)_{R}$ \\
\hline$Q$ & $f$ & $f$ & 0 \\
$V$ & $a d j$ & 1 & 1 \\
\hline$M$ & & $T_{A}$ & 0 \\
\hline
\end{tabular}

Table 6. A $4 d$ theory with $G_{c}=S P(2 N)$ and $2 N+2$ quarks exhibiting the chiral symmetry breaking.

\section{Chiral symmetry breaking for $G_{c}=S P(2 N)$}

Consider chiral symmetry breaking in a $\mathcal{N}=1$ SYM theory with the gauge group $S P(2 N)$. Let us start from the $s$-confining theory with $G_{c}=S P(2 N)$ and $2 N+4$ quarks studied in [44] with the identification of the number of flavors as $N_{f}=N+2$. Corresponding (electric) SCI is $[1,12]$

$$
\begin{gathered}
I_{E}=\frac{(p ; p)_{\infty}^{N}(q ; q)_{\infty}^{N}}{2^{N} N !} \int_{\mathbb{T}^{N}} \prod_{1 \leq i<j \leq N} \frac{1}{\Gamma\left(z_{i}^{ \pm 1} z_{j}^{ \pm 1} ; p, q\right)} \\
\times \prod_{j=1}^{N} \frac{\prod_{m=1}^{2 N+4} \Gamma\left(t_{m} z_{j}^{ \pm 1} ; p, q\right)}{\Gamma\left(z_{j}^{ \pm 2} ; p, q\right)} \frac{d z_{j}}{2 \pi \mathrm{i} z_{j}}
\end{gathered}
$$

where $\left|t_{m}\right|<1$ and the balancing condition reads $\prod_{m=1}^{2 N+4} t_{m}=p q$. The dual (magnetic) theory is described by colorless mesons forming the $T_{A}$-representation of $\mathrm{SU}(2 N+4)$ group with the index

$$
I_{M}=\prod_{1 \leq m<s \leq 2 N+4} \Gamma\left(t_{m} t_{s} ; p, q\right)
$$

The equality $I_{E}=I_{M}$ was suggested in [45] and proved in [10, 11]. As in the previous $G_{c}=\mathrm{SU}(N)$ case we integrate out two quark fields by restricting chemical potentials as, say, $t_{2 N+3} t_{2 N+c+4}=p q$. As a result, dependence on the parameters $t_{2 N+3}$ and $t_{2 N+4}$ disappears from $I_{E}$ which yields formally the index of the theory with $2 N+2$ chiral fields. For generic values of other fugacities, $I_{M}$ is equal to zero, but there are delta function singularities for a singular submanifold of fugacities. For the taken $S P(2 N)$-gauge group the conformal window where the general Seiberg duality is supposed to be valid has the form $3(N+1) / 2<$ $N_{f}<3(N+1)$, our duality corresponds to $N_{f}=N+1$ and lies outside this window.

A theory with $S P(2 N)$ gauge group and quantum modified moduli space was described in [44]. The matter content for corresponding electric and magnetic theories is presented in table 6 . The mesonic fields are composed as $M_{i j}=Q_{i} Q_{j}$, where the $S P(2 N)$ symplectic trace is assumed making the mesons gauge invariant. The quantum moduli space of vacua satisfies the constraint

$$
\operatorname{Pf} M=\Lambda^{2(N+1)},
$$

with some energy scale $\Lambda$ which breaks the conformal symmetry with appropriate consequences for interpreting our SCIs. 
Naively the electric theory has the $\mathrm{SU}(2(N+1))$ flavor group with $2 N+1$ independent fugacities. Corresponding fundamental representation character has the form

$$
\chi_{f, \mathrm{SU}(2 N+2)}(\underline{x})=\sum_{i=1}^{2 N+2} x_{i}
$$

where $x_{i}$ are fugacities for maximal torus generators of $\mathrm{SU}(2 N+2)$ restricted by the constraint $\prod_{i=1}^{2 N+2} x_{i}=1$. The chiral symmetry breaking reduces this naive flavor group to $S P(2 N)$. Therefore it is necessary to describe how the character (6.3) reduces to the fundamental representation character of $S P(2 N)$

$$
\chi_{f, S P(2 N+2)}(\underline{y})=\sum_{i=1}^{N+1}\left(y_{i}+y_{i}^{-1}\right),
$$

where $y_{1}, \ldots, y_{N+1}$ are maximal torus fugacities without constraints. Evidently, this can be done if one identifies half of $x_{i}$ variables with $y_{j}$ and forces the rest of $x_{i}$-variables to coincide with $y_{j}^{-1}$ (which resolves automatically the balancing condition). This observation hints that one should realize the constraints $x_{i} x_{j}=1, i \neq j$, for all possible splittings of $x_{i}$-variables into pairs.

In order to find the structure of $I_{E}$ in the case of chiral symmetry breaking, we set $t_{2 N+3} t_{2 N+c+4}=p q e^{\epsilon}$ in (6.1) and (6.2) and consider the limit $\epsilon \rightarrow 0$. Then, expression (6.2) contains the multiplier $\Gamma\left(p q e^{\epsilon} ; p, q\right)$ tending to zero which can be overpowered only by the poles of other elliptic gamma functions. Because now $\prod_{j=1}^{2 N+2} t_{j}=e^{-\epsilon}$, in the limit $\epsilon \rightarrow 0$ we can identify $t_{j}=x_{j}$. Originally, the equality $I_{E}=I_{M}$ was obtained for $\left|t_{i}\right|<1$ for all $i$, however it can be meromorphically continued to arbitrary values of the parameters. To test the singularities we multiply $I_{M}$ by an arbitrary holomorphic function $f\left(t_{1}, \ldots, t_{N+1}\right)$ weighted by a specific product of elliptic gamma functions and integrate over $t_{1}, \ldots, t_{N} \in \mathbb{T}$ :

$$
\int_{\mathbb{T}^{N}} \rho(\underline{t}) f\left(t_{1}, \ldots, t_{N+1}\right) I_{E} \prod_{k=1}^{N} \frac{d t_{k}}{2 \pi \mathrm{i} t_{k}}, \quad \rho(\underline{t})=\frac{1}{\prod_{1 \leq i<j \leq N+1} \Gamma\left(t_{i} t_{j} ; p, q\right)},
$$

where we assume that the balancing condition is resolved in favor of the variable $t_{N+1}$ :

$$
t_{N+1}=\frac{e^{-\epsilon}}{\prod_{k=1}^{N} t_{k} \prod_{l=1}^{N+1} t_{l+N+1}} .
$$

Multiplication of $I_{E}$ by $\rho(\underline{t})$ removes a number of singularities which are associated with the zero locus of $\rho(\underline{t})$. However, the latter singularities can be restored later on by the permutational symmetry in variables $t_{i}$. Replacing $I_{E}$ by $I_{M}$ we come to the expression

$$
\begin{aligned}
\Gamma\left(p q e^{\epsilon} ; p, q\right) & \prod_{N+2 \leq i<j \leq 2 N+2} \Gamma\left(t_{i} t_{j} ; p, q\right) \int_{\mathbb{T}^{N}} f\left(t_{1}, \ldots, t_{N+1}\right) \\
\times & \prod_{i=1}^{N} \prod_{j=N+2}^{2 N+2} \Gamma\left(t_{i} t_{j} ; p, q\right) \prod_{j=N+2}^{2 N+2} \Gamma\left(\frac{t_{j} e^{-\epsilon}}{\prod_{k=1}^{N} t_{k} \prod_{l=1}^{N+1} t_{l+N+1}} ; p, q\right)
\end{aligned}
$$




$$
\times \prod_{j=1}^{2 N+2} \Gamma\left(t_{j} t_{2 N+3}, \frac{p q e^{\epsilon} t_{j}}{t_{2 N+3}} ; p, q\right) \prod_{k=1}^{N} \frac{d t_{k}}{2 \pi \mathrm{i} t_{k}} .
$$

Consider singularities of the integrand near the integration contours. For $\epsilon>0$ we can take $\left|t_{j}\right|=e^{-\epsilon /(N+2)}<1, i=N+1, \ldots, 2 N+2$, so that in the limit $\epsilon \rightarrow 0$ one has $t_{i} \rightarrow \mathbb{T}$ for $i=1, \ldots, 2 N+2$. Let us take the absolute values of $t_{2 N+3}$ and $t_{2 N+4}$ sufficiently small, so that the poles of the elliptic gamma functions on the last line in (6.5) do not approach $\mathbb{T}$ and stay harmless. Then the relevant poles are

$$
\text { out : } \quad t_{i}=t_{j}^{-1}, \quad i=1, \ldots, N, \quad j=N+2, \ldots, 2 N+2,
$$

lying outside $\mathbb{T}$ and

$$
\text { in }: \quad \prod_{i=1}^{N} t_{i}=\frac{e^{-\epsilon} t_{j}}{\prod_{l=1}^{N+1} t_{l+N+1}}, \quad j=N+2, \ldots, 2 N+2,
$$

lying inside $\mathbb{T}$. Consider first the integral in $t_{1}$. There always exist such values of $t_{2}, \ldots, t_{N}$ that the in poles approach $\mathbb{T}$ from inside at the points $t_{1} \rightarrow t_{j}^{-1}, j=N+2, \ldots, 2 N+2$, and there emerge pinchings of $\mathbb{T}$ by in and out poles. These poles are simple provided $t_{j} \neq t_{k}, j \neq k$, which we assume. To deal with that we inflate a little all integration contours $\mathbb{T}$ and pick up the resides of all out poles. These residues have singularities of a similar structure and one can continue taking these "residues of residues" in $t_{2}, t_{3}$, etc until the last integration variable $t_{N}$. Considering the sequence of residues at $t_{i}=t_{N+1+i}^{-1}$, on the last step one obtains the diverging multiplier $\Gamma\left(t_{N+1} t_{2 N+2} ; p, q\right)=\Gamma\left(e^{-\epsilon} ; p, q\right)$ which cancels the vanishing factor $\Gamma\left(p q e^{\epsilon}\right)$. Similar situation holds for taking pole residues in any other possible order. In the limit $\epsilon \rightarrow 0$ only these highest order residues survive, since if one misses at least one residue in the intermediate step, no divergency is taking place and the corresponding term vanishes.

As a result, we obtain

$$
\begin{aligned}
\int_{\mathbb{T}^{N}} \frac{f\left(t_{1}, \ldots, t_{N+1}\right)}{\prod_{1 \leq i<j \leq N+1} \Gamma\left(t_{i} t_{j} ; p, q\right)} I_{E} \prod_{k=1}^{N} \frac{d t_{k}}{2 \pi \mathrm{i} t_{k}} \\
\quad \underset{\epsilon \rightarrow 0}{=} \frac{\prod_{N+2 \leq i<j \leq 2 N+2} \Gamma\left(t_{i} t_{j}, t_{i}^{-1} t_{j}, t_{i} t_{j}^{-1} ; p, q\right)}{(p ; p)_{\infty}^{N}(q ; q)_{\infty}^{N}} \sum_{\tilde{t}_{j}} f\left(\tilde{t}_{N+2}^{-1}, \ldots, \tilde{t}_{2 N+2}^{-1}\right),
\end{aligned}
$$

where $\left(\tilde{t}_{N+2}, \ldots, \tilde{t}_{2 N+2}\right)=\mathcal{P}\left(t_{N+2}, \ldots, t_{2 N+2}\right)$ is any permutation of the parameters.

Denote now $t_{j}=e^{2 \pi \mathrm{i} \phi_{j}}$ and use real variables $\phi_{j}$ to write $I_{E}$ as a distribution. The full set of singularities of $I_{E}$, which was partially reduced after multiplication by $\rho(\underline{t})$, is restored from complete $S_{2 N+2}$-group permutational symmetry of the index.

Because of the balancing condition $\sum_{i=1}^{2 N+2} \phi_{i}=0$ we have $2 N+1$ independent variables $\phi_{i}$. Assume as before that $\phi_{N+1}$ is fixed by other parameters. Consider an arbitrary split of the set $\Phi=\left(\phi_{1}, \ldots, \phi_{2 N+2}\right)$ into two $(N+1)$-term groups $\Phi_{1}=\left(\tilde{\phi}_{1}, \ldots, \tilde{\phi}_{N}, \tilde{\phi}_{N+1}=\phi_{N+1}\right)$ and $\Phi_{2}=\left(\tilde{\phi}_{N+2}, \ldots, \tilde{\phi}_{2 N+2}\right)$. Then we pair parameters in $i$-th position, $i=1, \ldots, N$, in these groups and impose the constraints $\tilde{\phi}_{i}+\tilde{\phi}_{N+1+i}=0$. Because of the balancing 
condition, the remaining pair of parameters satisfies the constraint $\phi_{N+1}+\phi_{2 N+2}=0$ automatically. Now we form a sum of products of delta functions

$$
\sum_{S_{N+1}\left(\Phi_{2}\right)} \prod_{i=1}^{N} \delta\left(\tilde{\phi}_{i}+\tilde{\phi}_{N+1+i}\right),
$$

where the sum goes over all possible $(N+1)$ ! permutations of elements of the set $\Phi_{2}$. Evidently this sum is also symmetric under $N$ ! permutations of the elements in the first set $\Phi_{1}$ and $2^{N}$ permutations of $\tilde{\phi}_{i}$ with $\tilde{\phi}_{N+1+i}$ belonging to different sets. Using this auxiliary building block, we can write the final relation for our SCIs in the following form

$$
\begin{aligned}
& I_{E}= \frac{(p ; p)_{\infty}^{N}(q ; q)_{\infty}^{N}}{2^{N} N !} \int_{\mathbb{T}_{d}^{N}} \prod_{1 \leq i<j \leq N} \frac{1}{\Gamma\left(z_{i}^{ \pm 1} z_{j}^{ \pm 1} ; p, q\right)} \\
& \times \prod_{j=1}^{N} \frac{\prod_{i=1}^{2 N+2} \Gamma\left(e^{2 \pi \mathrm{i} \phi_{i}} z_{j}^{ \pm 1} ; p, q\right)}{\Gamma\left(z_{j}^{ \pm 2} ; p, q\right)} \frac{d z_{j}}{2 \pi \mathrm{i} z_{j}}=I_{M}=\frac{1}{(p ; p)_{\infty}^{N}(q ; q)_{\infty}^{N}} \\
& \times \sum_{\left(\Phi_{1} \cup \Phi_{2}\right) / S_{2}^{N}} \prod_{1 \leq i<j \leq N+1} \Gamma\left(e^{2 \pi \mathrm{i}\left( \pm \tilde{\phi}_{i} \pm \tilde{\phi}_{j}\right)} ; p, q\right) \sum_{S_{N+1}\left(\Phi_{2}\right)} \prod_{i=1}^{N} \delta\left(\tilde{\phi}_{i}+\tilde{\phi}_{N+1+i}\right),
\end{aligned}
$$

where the first sum goes over all possible splits of $\Phi$ into $\Phi_{1}$ and $\Phi_{2}$ modulo $2^{N}$ permutation of the paired parameters. In the electric SCI the integration contour $\mathbb{T}_{d}$ is a deformation of $\mathbb{T}$ such that it separates sequences of the integrand poles converging to zero from their reciprocals, i.e. $e^{2 \pi \mathrm{i} \phi_{i}}$ lie inside $\mathbb{T}_{d}$ and $e^{-2 \pi \mathrm{i} \phi_{i}}$ are outside $\mathbb{T}_{d}$.

It is not difficult to see that one can replace fixed $\phi_{N+1}$ by any other parameter and it will give the same result, i.e. the final answer is $S_{2 N+2}$-group symmetric. Therefore, one may replace both sums in (6.6) by a single sum over all permutations of $\phi_{i}, i=1, \ldots, 2 N+2$, and divide it by $(2 N+2) N ! 2^{N}$ counting the number of equal terms. For $N=1$ relation (6.6) coincides with the equality of SCIs considered in section 3.

We conclude that the electric SCI is non-vanishing only on the support of indicated products of delta functions. For each such product one has the reduction of the character of fundamental representations of $\mathrm{SU}(2 N+2)$-group down to the corresponding character of $S P(2 N+2)$-group, as prescribed by the chiral symmetry breaking and naive recipe of building SCIs. On the dual side the products of elliptic gamma functions coincide with the $\phi_{i}$-dependent part of SCIs for free meson fields forming the $T_{A}$-representation of $S P(2 N+2)$-group with the character

$$
\chi_{T_{A}, S P(2 N+2)}=\sum_{1 \leq i<j \leq N+1} \sum_{\mu= \pm 1, \nu= \pm 1} e^{2 \pi \mathrm{i}\left(\mu \phi_{i}+\nu \phi_{j}\right)}+N .
$$

The formal prescription for building SCIs would yield from the constant $N$ the diverging factor $\Gamma(1 ; p, q)^{N}$, which in our rigorous consideration is replaced by the product of delta functions divided by $(p ; p)_{\infty}^{N}(q ; q)_{\infty}^{N}$. We see again that for theories with chiral symmetry breaking the standard recipe of constructing SCIs requires a careful modification.

An interesting situation arises in the rank 3 case, i.e. for the $S P(6)$-gauge group with 8 chiral superfields. In this case the multiple duality phenomenon takes place, which follows 
from the considerations of [12] for the special value of the corresponding U(1)-group fugacity $t=\sqrt{p q}$. These theories lie outside of the conformal window and their content was described in [13]. This means that there are three more interacting field theories with the same gauge group and 8 quarks showing the chiral symmetry breaking whose "superconformal" indices should coincide with the one for our electric/magnetic theory. However, the expression (6.6) does not satisfy this property - it is not invariant under the transformation of fugacities from $W\left(E_{7}\right)$-group accompanied by multiplication of the index by certain products of elliptic gamma functions [12]. Under these transformations new combinations of the delta functions emerge which were forbidden by our constraints on the parameters, i.e. a more careful extended analysis of the situation is needed which we postpone to a later time.

As to such extended symmetries for indices we mention that the considerations of $W\left(E_{7}\right)$ and $W\left(E_{6}\right)$-invariant SCIs in [30,31] should be reducible to one more level down to the $W\left(F_{4}\right)$-symmetric instance. Namely, there should exist some combination of fugacities after multiplication by which the combination of delta functions in $I_{M}$ for $N_{f}=N=2$ or for more general theories of [12] should be invariant with respect to the Weyl group $W\left(F_{4}\right)$. Again a more detailed investigation of emerging singularities may be required and the consideration of such a possibility lies beyond the scope of the present work.

\section{$7 \quad 3 d$ theories with chiral symmetry breaking}

Recently there was a breakthrough in investigation of $3 d$ supersymmetric field theories due to the calculation of partition functions (see, e.g. [46-50]). As shown in [51] (see also $[52,53]) 4 d$ superconformal indices can be reduced to $3 d$ partition functions which yields a reduction of the related $4 d$ Seiberg dualities to $3 d$ SYM or CS theory dualities. To our knowledge this scheme is the most efficient way of producing $3 d$ dualities after appropriate amendment of the superpotentials [54].

To realize the $4 d / 3 d$ reduction in the simplest $s$-confining theory one considers a special limit of the elliptic beta integral. First one parametrizes the variables as

$$
p=e^{2 \pi \mathrm{i} r \omega_{1}}, \quad q=e^{2 \pi \mathrm{i} r \omega_{2}}, \quad s_{j}=e^{2 \pi \mathrm{i} r \phi_{j}}, \quad z=e^{2 \pi \mathrm{i} r u}
$$

and then takes the limit $r \rightarrow 0$. To simplify the integrals one uses the Ruijsenaars limit for elliptic gamma function

$$
\Gamma\left(e^{2 \pi \mathrm{i} r u} ; e^{2 \pi \mathrm{i} r \omega_{1}}, e^{2 \pi \mathrm{i} r \omega_{2}}\right) \underset{r \rightarrow 0}{=} e^{-\pi \mathrm{i}\left(2 z-\omega_{1}-\omega_{2}\right) / 12 r \omega_{1} \omega_{2}} \gamma^{(2)}\left(u ; \omega_{1}, \omega_{2}\right),
$$

where

$$
\gamma^{(2)}\left(u ; \omega_{1}, \omega_{2}\right)=e^{-\frac{\pi \mathrm{i}}{2} B_{2,2}\left(u ; \omega_{1}, \omega_{2}\right)} \frac{\left(e^{2 \pi \mathrm{i}\left(u-\omega_{2}\right) / \omega_{1}} ; e^{-2 \pi \mathrm{i} \omega_{2} / \omega_{1}}\right)_{\infty}}{\left(e^{2 \pi \mathrm{i} u / \omega_{2}} ; e^{2 \pi \mathrm{i} \omega_{1} / \omega_{2}}\right)_{\infty}}
$$

is the hyperbolic gamma function and $B_{2,2}(u ; \omega)$ is the second order Bernoulli polynomial,

$$
B_{2,2}(u ; \omega)=\frac{u^{2}}{\omega_{1} \omega_{2}}-\frac{u}{\omega_{1}}-\frac{u}{\omega_{2}}+\frac{\omega_{1}}{6 \omega_{2}}+\frac{\omega_{2}}{6 \omega_{1}}+\frac{1}{2}
$$

The following conventions are used below $\gamma^{(2)}(a, b ; \omega):=\gamma^{(2)}(a ; \omega) \gamma^{(2)}(b ; \omega)$ and $\gamma^{(2)}(a \pm$ $u ; \omega):=\gamma^{(2)}(a+u ; \omega) \gamma^{(2)}(a-u ; \omega)$. The function $\gamma^{(2)}\left(u ; \omega_{1}, \omega_{2}\right)$ has poles at $u=-n \omega_{1}-m \omega_{2}$ 
for $n, m \in \mathbb{Z}_{\geq 0}$, zeros at $u=n \omega_{1}+m \omega_{2}$ for $n, m \in \mathbb{Z}_{>0}$ and satisfies the inversion relation $\gamma^{(2)}\left(u, \omega_{1}+\omega_{2}-u ; \omega_{1}, \omega_{2}\right)=1$.

Taking the limit $r \rightarrow 0$ along the negative imaginary axis and assuming that $\operatorname{Re}\left(\omega_{1}\right)$, $\operatorname{Re}\left(\omega_{2}\right)>0$ one gets the following reduction of the electric SCI (up to some diverging factor, see e.g. [51])

$$
I_{E}^{r e d}=\int_{-\mathrm{i} \infty}^{\mathrm{i} \infty} \frac{\prod_{k=1}^{6} \gamma^{(2)}\left(\phi_{k} \pm u ; \omega_{1}, \omega_{2}\right)}{\gamma^{(2)}\left( \pm 2 u ; \omega_{1}, \omega_{2}\right)} \frac{d u}{2 \mathrm{i} \sqrt{\omega_{1} \omega_{2}}}
$$

where the balancing condition has the form $\sum_{k=1}^{6} \phi_{k}=\omega_{1}+\omega_{2}$ and the integration contour separates sequences of poles going to infinity on the right- and left-hand sides of the imaginary axis. The magnetic theory SCI reduces to (up to the same diverging factor as in $I_{E}$ )

$$
I_{M}^{r e d}=\prod_{1 \leq j<k \leq 6} \gamma^{(2)}\left(\phi_{j}+\phi_{k} ; \omega_{1}, \omega_{2}\right) .
$$

Impose now the constraint $\phi_{5}+\phi_{6}=\omega_{1}+\omega_{2}+\epsilon$ and take the limit $\epsilon \rightarrow 0$. The limiting balancing condition takes the form $\phi_{1}+\ldots+\phi_{4}=0$, and we can take all $\phi_{i}$ as purely imaginary numbers, $\phi_{m}=\mathrm{i} g_{m}, g_{m} \in \mathbb{R}$. Let us apply the scheme of consideration of singularities of the previous section in the present setting using the relation

$$
2 \pi \mathrm{i} \lim _{g \rightarrow 0} g \gamma^{(2)}\left(\mathrm{i} g ; \omega_{1}, \omega_{2}\right)=\sqrt{\omega_{1} \omega_{2}}
$$

for computing the residues. As a result we obtain the expressions

$$
I_{E}^{r e d}=\int_{\mathcal{C}} \frac{\prod_{m=1}^{4} \gamma^{(2)}\left(\mathrm{i} g_{m} \pm u ; \omega_{1}, \omega_{2}\right)}{\gamma^{(2)}\left( \pm 2 u ; \omega_{1}, \omega_{2}\right)} \frac{d u}{2 \mathrm{i} \sqrt{\omega_{1} \omega_{2}}}
$$

where the integration contour is an infinitesimal deformation of the imaginary axis such that the poles $u=\mathrm{i} g_{j}+n \omega_{1}+m \omega_{2}, n, m \in \mathbb{Z}_{\geq 0}$, lie to the right of $\mathcal{C}$ and their reciprocals $u \rightarrow-u$ are to the left of $\mathcal{C}$. The magnetic theory yields

$$
\begin{array}{r}
I_{M}^{r e d}=\sqrt{\omega_{1} \omega_{2}}\left(\gamma ^ { ( 2 ) } \left( \pm \mathrm{i} g_{1}\right.\right. \\
\left. \pm \mathrm{i} g_{2} ; \omega_{1}, \omega_{2}\right)\left(\delta\left(g_{1}+g_{3}\right)+\delta\left(g_{1}+g_{4}\right)\right) \\
\left.+\gamma^{(2)}\left( \pm \mathrm{i} g_{2} \pm \mathrm{i} g_{3} ; \omega_{1}, \omega_{2}\right) \delta\left(g_{1}+g_{2}\right)\right),
\end{array}
$$

where $\delta(g)$ is the standard (non-periodic) delta function. The equality $I_{E}^{\text {red }}=I_{M}^{\text {red }}$ expresses coincidence of partitions functions of two $\mathcal{N}=23 d$ theories whose matter content is the same as in table 3 with the replacement $4 d \rightarrow 3 d$. This example of chiral symmetry breaking corresponds to the $N_{f}=N=2$ case duality in the considerations of [54].

Denote now

$$
g_{1}=\mu+x, \quad g_{1}=\mu-x, \quad g_{3}=-\mu+y, \quad g_{4}=-\mu-y
$$

and take the limit $\mu \rightarrow+\infty$. Using the asymptotic properties of the hyperbolic gamma function for $\operatorname{Im}\left(\omega_{1} / \omega_{2}\right)>0$,

$$
\lim _{u \rightarrow \infty} e^{\frac{\pi \mathrm{i}}{2} B_{2,2}(u ; \omega)} \gamma^{(2)}\left(u ; \omega_{1}, \omega_{2}\right)=1, \quad \text { for } \arg \omega_{1}<\arg u<\arg \omega_{2}+\pi,
$$




\begin{tabular}{|c|c|c|c|}
\hline & $\mathrm{U}(1)$ & $\mathrm{SU}(2)$ & $\mathrm{U}(1)_{R}$ \\
\hline$Q$ & $f$ & $f$ & 0 \\
$\tilde{Q}$ & $\bar{f}$ & $f$ & 0 \\
$V$ & $a d j$ & 1 & 1 \\
\hline$q$ & & $a d j$ & 0 \\
\hline
\end{tabular}

Table 7. A $3 d$ theory with the chiral symmetry breaking.

$$
\lim _{u \rightarrow \infty} e^{-\frac{\pi \mathrm{i}}{2} B_{2,2}(u ; \omega)} \gamma^{(2)}\left(u ; \omega_{1}, \omega_{2}\right)=1, \quad \text { for } \arg \omega_{1}-\pi<\arg u<\arg \omega_{2}
$$

we can see that $I_{E}^{r e d}=\gamma Z_{E}$, where $\gamma$ is the diverging factor $\gamma=\exp \left(-2 \pi \mu\left(\omega_{1}^{-1}+\omega_{2}^{-1}\right)\right)$, and

$$
Z_{E}=e^{\pi \mathrm{i}\left(x^{2}-y^{2}\right) / \omega_{1} \omega_{2}} \int_{-\mathrm{i} \infty}^{\mathrm{i} \infty} \gamma^{(2)}\left( \pm \mathrm{i} x-u, \pm \mathrm{i} y+u ; \omega_{1}, \omega_{2}\right) \frac{d u}{2 \mathrm{i} \sqrt{\omega_{1} \omega_{2}}} .
$$

Similarly, $I_{M}^{r e d}=\gamma Z_{M}$, where

$$
Z_{M}=\sqrt{\omega_{1} \omega_{2}} \gamma^{(2)}\left( \pm 2 \mathrm{i} x ; \omega_{1}, \omega_{2}\right)(\delta(x-y)+\delta(x+y)) .
$$

There are only two delta functions since the argument of the third one goes to infinity, $g_{1}+g_{2}=2 \mu \rightarrow+\infty$, i.e. it does not give contributions. The multiplier $e^{\pi \mathrm{i}\left(x^{2}-y^{2}\right) / \omega_{1} \omega_{2}}$ can be dropped in $Z_{E}$, since $Z_{E}$ vanishes for $x \neq \pm y$.

The identity $Z_{E}=Z_{M}$ expresses the equality of partition functions of two dual $3 d$ $\mathcal{N}=2$ supersymmetric field theories described in [55]. The (real) electric theory has $\mathrm{U}(1)$ gauge group and $N_{f}=2$ chiral fields with the broken $\mathrm{U}(1)_{A}$ symmetry and naive $\mathrm{SU}(2)_{l} \times \mathrm{SU}(2)_{r}$ flavor group broken to the diagonal subgroup $\mathrm{SU}(2)$. The magnetic theory has no local gauge group symmetry and consists of only confined meson fields. The matter content of these dual theories is presented in table 7 .

Again, the original recipe of building $3 d$ partition functions [46] requires a modification for theories exhibiting chiral symmetry breaking - in the electric part the contour of integration should be chosen appropriately and in the magnetic part contributions of constant terms in the characters of representations yielding $\gamma\left(0 ; \omega_{1}, \omega_{2}\right)$ should be replaced by delta functions. In the above example, the magnetic theory meson fields form the adjoint representation of $\mathrm{SU}(2)$ flavor group with the character $x^{2}+x^{-2}+1$. The latter constant "1" formally yields in $Z_{M}$ the factor $\gamma\left(0 ; \omega_{1}, \omega_{2}\right)$, which should be replaced in reality by $\sqrt{\omega_{1} \omega_{2}}(\delta(x-y)+\delta(x+y))$, where $x$ and $y$ are fugacities of the naive flavor group $\mathrm{SU}(2)_{l} \times \mathrm{SU}(2)_{r}$. We would like to stress that our interpretation of vanishing partition functions differs from the one made in [56] where corresponding partition functions were equal to zero due to the mass parameters lying in the general position. Evidently one can proceed in a similar manner and consider other examples of $4 d$ dual theories with chiral symmetry breaking and reduce them to $3 d$ partners exhibiting similar phenomenon [54]. In particular, it is possible to consider $3 d$ partners of the $4 d$ theories described in [13] and find multiple dualities with this property. 


\section{Conclusion}

To conclude, as a continuation of our previous considerations of the relation between properties of elliptic hypergeometric integrals and superconformal indices [12]-[18], we have described how to compute these indices in the theories with chiral symmetry breaking. The original prescription [5-7] needs modification in this case and the theory of elliptic hypergeometric integrals yields the required recipe. The chiral symmetry breaking mechanism is reflected in the appearance of delta functions in the indices of original theories with naive chiral symmetry such that their support yields constraints on the fugacities describing the quantum deformed moduli spaces with real symmetries. This mechanism survives in the $4 d \rightarrow 3 d$ reduction simply by the reduction of corresponding $4 d$ superconformal indices to $3 d$ partition functions.

The results from the analysis of SCIs or partition functions allow one to find easily the field content of the theories. However, a deeper physical investigation of our results is needed. Namely, the physical meaning of the index in this situation should be reconsidered with an explanation of the emergence of delta functions from the localization procedure.

\section{Acknowledgments}

This work was partially supported by the RFBR grant no. 12-01-00242 and the HeisenbergLandau program. We would like to thank organizers of the workshop "Aspects of conformal and superconformal field theories" at the Cambridge University in April 2012, where preliminary results of this paper were reported. We are indebted also to H. Osborn and N. Seiberg for useful comments and to the anonymous referee for constructive remarks.

Open Access. This article is distributed under the terms of the Creative Commons Attribution License (CC-BY 4.0), which permits any use, distribution and reproduction in any medium, provided the original author(s) and source are credited.

\section{References}

[1] F.A. Dolan and H. Osborn, Applications of the superconformal index for protected operators and q-hypergeometric identities to $\mathcal{N}=1$ dual theories, Nucl. Phys. B $\mathbf{8 1 8}$ (2009) 137 [arXiv: 0801.4947] [INSPIRE].

[2] V.P. Spiridonov, On the elliptic beta function, Usp. Mat. Nauk 56 (2001) 181 [Russ. Math. Surv. 56 (2001) 185].

[3] V.P. Spiridonov, Theta hypergeometric integrals, Alg. Analiz. 15 (2003) 161 [St. Petersburg Math. J. 15 (2004) 929] [math.CA/0303205].

[4] V.P. Spiridonov, Essays on the theory of elliptic hypergeometric functions, Usp. Mat. Nauk 63 (2008) 3 [Russ. Math. Surv. 63 (2008) 405] [arXiv:0805.3135].

[5] J. Kinney, J.M. Maldacena, S. Minwalla and S. Raju, An index for 4 dimensional super conformal theories, Commun. Math. Phys. 275 (2007) 209 [hep-th/0510251] [INSPIRE].

[6] C. Romelsberger, Counting chiral primaries in $\mathcal{N}=1, D=4$ superconformal field theories, Nucl. Phys. B 747 (2006) 329 [hep-th/0510060] [INSPIRE]. 
[7] C. Romelsberger, Calculating the superconformal index and Seiberg duality, arXiv:0707.3702 [INSPIRE].

[8] G. Festuccia and N. Seiberg, Rigid supersymmetric theories in curved superspace, JHEP 06 (2011) 114 [arXiv:1105.0689] [INSPIRE].

[9] N. Seiberg, Electric-magnetic duality in supersymmetric non-Abelian gauge theories, Nucl. Phys. B 435 (1995) 129 [hep-th/9411149] [INSPIRE].

[10] E.M. Rains, Transformations of elliptic hypergeometric integrals, Ann. Math. 171 (2010) 169.

[11] V.P. Spiridonov, Short proofs of the elliptic beta integrals, Ramanujan J. 13 (2007) 265 [math. CA/0408369].

[12] V.P. Spiridonov and G.S. Vartanov, Superconformal indices for $\mathcal{N}=1$ theories with multiple duals, Nucl. Phys. B 824 (2010) 192 [arXiv:0811.1909] [InSPIRE].

[13] V.P. Spiridonov and G.S. Vartanov, Supersymmetric dualities beyond the conformal window, Phys. Rev. Lett. 105 (2010) 061603 [arXiv:1003.6109] [INSPIRE].

[14] V.P. Spiridonov and G.S. Vartanov, Elliptic hypergeometry of supersymmetric dualities, Commun. Math. Phys. 304 (2011) 797 [arXiv:0910.5944] [INSPIRE].

[15] V.P. Spiridonov and G.S. Vartanov, Elliptic hypergeometry of supersymmetric dualities II. Orthogonal groups, knots and vortices, Commun. Math. Phys. 325 (2014) 421 [arXiv:1107.5788] [INSPIRE].

[16] V.P. Spiridonov and G.S. Vartanov, Superconformal indices of $\mathcal{N}=4$ SYM field theories, Lett. Math. Phys. 100 (2012) 97 [arXiv: 1005.4196] [INSPIRE].

[17] G.S. Vartanov, On the ISS model of dynamical SUSY breaking, Phys. Lett. B 696 (2011) 288 [arXiv: 1009.2153] [INSPIRE].

[18] V.P. Spiridonov and G.S. Vartanov, Elliptic hypergeometric integrals and 't Hooft anomaly matching conditions, JHEP 06 (2012) 016 [arXiv: 1203.5677] [INSPIRE].

[19] A. Gadde, E. Pomoni, L. Rastelli and S.S. Razamat, S-duality and $2 d$ topological QFT, JHEP 03 (2010) 032 [arXiv:0910.2225] [INSPIRE].

[20] A. Gadde, L. Rastelli, S.S. Razamat and W. Yan, The superconformal index of the $E_{6} S C F T$, JHEP 08 (2010) 107 [arXiv: 1003.4244] [INSPIRE].

[21] A. Gadde, L. Rastelli, S.S. Razamat and W. Yan, Gauge theories and Macdonald polynomials, Commun. Math. Phys. 319 (2013) 147 [arXiv:1110.3740] [INSPIRE].

[22] M. Sudano, The Romelsberger index, Berkooz deconfinement and infinite families of Seiberg duals, JHEP 05 (2012) 051 [arXiv: 1112.2996] [INSPIRE].

[23] B.I. Zwiebel, Charging the superconformal index, JHEP 01 (2012) 116 [arXiv:1111.1773] [INSPIRE].

[24] Y. Nakayama, $4 D$ and $2 D$ superconformal index with surface operator, JHEP 08 (2011) 084 [arXiv: 1105.4883] [INSPIRE].

[25] D. Gaiotto, L. Rastelli and S.S. Razamat, Bootstrapping the superconformal index with surface defects, JHEP 01 (2013) 022 [arXiv:1207.3577] [INSPIRE].

[26] T. Dimofte, D. Gaiotto and S. Gukov, 3-manifolds and 3d indices, arXiv:1112.5179 [INSPIRE]. 
[27] D. Gang, E. Koh and K. Lee, Line operator index on $S^{1} \times S^{3}$, JHEP 05 (2012) 007 [arXiv:1201.5539] [INSPIRE].

[28] D. Gang, E. Koh and K. Lee, Superconformal index with duality domain wall, JHEP 10 (2012) 187 [arXiv:1205.0069] [INSPIRE].

[29] V.P. Spiridonov, Elliptic beta integrals and solvable models of statistical mechanics, Contemp. Math. 563 (2012) 181 [arXiv:1011.3798] [INSPIRE].

[30] T. Dimofte and D. Gaiotto, An E 7 surprise, JHEP 10 (2012) 129 [arXiv:1209.1404] [INSPIRE].

[31] I. Gahramanov and G. Vartanov, Extended global symmetries for $4 D \mathcal{N}=1 S Q C D$ theories, J. Phys. A 46 (2013) 285403 [arXiv:1303.1443] [InSPIRE].

[32] N. Seiberg, Exact results on the space of vacua of four-dimensional SUSY gauge theories, Phys. Rev. D 49 (1994) 6857 [hep-th/9402044] [INSPIRE].

[33] N.I. Muskhelishvili, Singular integral equations, Springer, Germany (1977).

[34] B. Grinstein and D.R. Nolte, Systematic study of theories with quantum modified moduli, Phys. Rev. D 57 (1998) 6471 [hep-th/9710001] [INSPIRE].

[35] B. Grinstein and D.R. Nolte, Systematic study of theories with quantum modified moduli. 2, Phys. Rev. D 58 (1998) 045012 [hep-th/9803139] [INSPIRE].

[36] V.P. Spiridonov, Continuous biorthogonality of the elliptic hypergeometric function, Alg. Analiz. 20 (2008) 155 [St. Petersburg Math. J. 20 (2009) 791] [arXiv:0801.4137].

[37] C. Csáki, M. Schmaltz and W. Skiba, A systematic approach to confinement in $\mathcal{N}=1$ supersymmetric gauge theories, Phys. Rev. Lett. 78 (1997) 799 [hep-th/9610139] [INSPIRE].

[38] C. Csáki, M. Schmaltz and W. Skiba, Confinement in $\mathcal{N}=1$ SUSY gauge theories and model building tools, Phys. Rev. D 55 (1997) 7840 [hep-th/9612207] [INSPIRE].

[39] V.P. Spiridonov, A Bailey tree for integrals, Teor. Mat. Fiz. 139 (2004) 104] [Theor. Math. Phys. 139 (2004) 536] [math.CA/0312502].

[40] V.P. Spiridonov and S.O. Warnaar, Inversions of integral operators and elliptic beta integrals on root systems, Adv. Math. 207 (2006) 91.

[41] S.E. Derkachov and V.P. Spiridonov, Yang-Baxter equation, parameter permutations and the elliptic beta integral, Usp. Mat. Nauk 68 (2013) 59 [Russ. Math. Surv. 68 (2013) 1027] [arXiv: 1205.3520] [INSPIRE].

[42] N.M. Davies, T.J. Hollowood, V.V. Khoze and M.P. Mattis, Gluino condensate and magnetic monopoles in supersymmetric gluodynamics, Nucl. Phys. B 559 (1999) 123 [hep-th/9905015] [INSPIRE].

[43] K. Konishi and A. Ricco, Calculating gluino condensates in $\mathcal{N}=1$ SYM from Seiberg-Witten curves, Phys. Lett. B 570 (2003) 118 [hep-th/0306128] [INSPIRE].

[44] K.A. Intriligator and P. Pouliot, Exact superpotentials, quantum vacua and duality in supersymmetric $\mathrm{SP}\left(N_{c}\right)$ gauge theories, Phys. Lett. B 353 (1995) 471 [hep-th/9505006] [INSPIRE].

[45] J.F. van Diejen and V.P. Spiridonov, Elliptic Selberg integrals, Internat. Math. Res. Notices 20 (2001) 1083. 
[46] A. Kapustin, B. Willett and I. Yaakov, Exact results for Wilson loops in superconformal Chern-Simons theories with matter, JHEP 03 (2010) 089 [arXiv: 0909.4559] [INSPIRE].

[47] N. Hama, K. Hosomichi and S. Lee, Notes on SUSY gauge theories on three-sphere, JHEP 03 (2011) 127 [arXiv: 1012.3512] [INSPIRE].

[48] N. Hama, K. Hosomichi and S. Lee, SUSY gauge theories on squashed three-spheres, JHEP 05 (2011) 014 [arXiv:1102.4716] [INSPIRE].

[49] F. Benini, C. Closset and S. Cremonesi, Comments on 3d Seiberg-like dualities, JHEP 10 (2011) 075 [arXiv:1108.5373] [INSPIRE].

[50] V. Niarchos, Seiberg dualities and the 3d/4d connection, JHEP 07 (2012) 075 [arXiv:1205.2086] [INSPIRE].

[51] F.A.H. Dolan, V.P. Spiridonov and G.S. Vartanov, From 4d superconformal indices to $3 d$ partition functions, Phys. Lett. B 704 (2011) 234 [arXiv:1104.1787] [INSPIRE].

[52] A. Gadde and W. Yan, Reducing the $4 d$ index to the $S^{3}$ partition function, JHEP 12 (2012) 003 [arXiv:1104.2592] [INSPIRE].

[53] Y. Imamura, Relation between the $4 d$ superconformal index and the $S^{3}$ partition function, JHEP 09 (2011) 133 [arXiv:1104.4482] [INSPIRE].

[54] O. Aharony, S.S. Razamat, N. Seiberg and B. Willett, $3 d$ dualities from $4 d$ dualities, JHEP 07 (2013) 149 [arXiv: 1305.3924] [INSPIRE].

[55] O. Aharony, A. Hanany, K.A. Intriligator, N. Seiberg and M.J. Strassler, Aspects of $\mathcal{N}=2$ supersymmetric gauge theories in three-dimensions, Nucl. Phys. B 499 (1997) 67 [hep-th/9703110] [INSPIRE].

[56] T. Morita and V. Niarchos, F-theorem, duality and SUSY breaking in one-adjoint Chern-Simons-matter theories, Nucl. Phys. B 858 (2012) 84 [arXiv:1108.4963] [InSPIRE]. 